Old Dominion University

ODU Digital Commons

\title{
Numerical Simulations of Capsule Deformation Using a Dual Time-Stepping Lattice Boltzmann Method
}

\author{
Charles Armstrong \\ Old Dominion University, carmstro@odu.edu \\ Yan Peng \\ Old Dominion University, ypeng@odu.edu
}

Follow this and additional works at: https://digitalcommons.odu.edu/mathstat_fac_pubs

Part of the Biological and Chemical Physics Commons, Fluid Dynamics Commons, and the Mathematics Commons

\section{Original Publication Citation}

Armstrong, C., \& Peng, Y. (2021). Numerical simulations of capsule deformation using a dual timestepping lattice Boltzmann method. Physical Review E, 103(2), 1-17, Article 023309 https://doi.org/ 10.1103/PhysRevE.103.023309

This Article is brought to you for free and open access by the Mathematics \& Statistics at ODU Digital Commons. It has been accepted for inclusion in Mathematics \& Statistics Faculty Publications by an authorized administrator of ODU Digital Commons. For more information, please contact digitalcommons@odu.edu. 


\title{
Numerical simulations of capsule deformation using a dual time-stepping lattice Boltzmann method
}

\author{
Charles Armstrong $\odot$ and Yan Peng $\odot$ \\ Department of Mathematics and Statistics, Old Dominion University, Norfolk, Virginia 23529, USA
}

(Received 6 July 2020; revised 22 January 2021; accepted 10 February 2021; published 26 February 2021)

\begin{abstract}
In this work a quasisteady, dual time-stepping lattice Boltzmann method is proposed for simulation of capsule deformation. At each time step the steady-state lattice Boltzmann equation is solved using the full approximation storage multigrid scheme for nonlinear equations. The capsule membrane is modeled as an infinitely thin shell suspended in an ambient fluid domain with the fluid structure interaction computed using the immersed boundary method. A finite element method is used to compute the elastic forces exerted by the capsule membrane. Results for a wide range of parameters and initial configurations are presented. The proposed method is found to reduce the computational time by a factor of ten.
\end{abstract}

DOI: 10.1103/PhysRevE.103.023309

\section{INTRODUCTION}

Capsules are elastic, fluid-filled membranes enclosing a Newtonian fluid, which serve as a useful model for biological and synthetic membranes. One prominent application of capsules has been to model the material properties of red blood cells. A number of experiments have been conducted on suspensions of red blood cells in a shear flow (e.g., Refs. [1-4]). As such, shear-induced capsule deformation has been the subject of extensive mathematical modeling.

Early analytical work was conducted by Barthés-Biesel [5] and Barthés-Biesel and Rallison [6] who used perturbation theory to predict the deformation of spherical capsules in shear flow in the limit of small deformations. Keller and Skalak [7] studied the behavior of rigid two dimensional ellipsoids in shear flow. This work was later extended to ellipsoids with elastic [8] and viscoelastic [9] membranes.

Because of the complex dynamics associated with capsule deformation, numerical studies have also drawn interest from researchers. Early numerical studies include the boundary element method (BEM) simulations done by Pozrikidis $[10,11]$ and Ramanujan and Pozrikidis [12] and the three dimensional immersed boundary method (IBM) [13] simulations reported by Eggleton and Popel in [14]. As computing power has progressed the body of research on capsule simulations of red blood cells has grown considerably, including BEM simulations in [15-20] and front-tracking and IBM simulations in [21-28].

In this work we propose a quasisteady, dual time-stepping (DTS) scheme that couples the multigrid lattice Boltzmann method (LBM) [29] to an IBM method to reduce the computational time required to simulate flow-induced capsule deformation at low Reynolds numbers. The fluid structure algorithm used here is similar to the LBM-IBM implementations discussed in Refs. [22,30,31]. The elasticity of the membrane is modeled using the finite element method proposed by Charrier et al. [32], where a constitutive law determines the membrane response to in-plane tensions. The capsule's bending resistance is described by the Helfrich bending functional $[33,34]$ and viscosity jumps across the membrane surface are handled using the front-tracking method proposed by Zhang et al. [35].

For LBM-IBM simulations of capsule deformation the resolution of the fluid velocity plays an outsized role in the accuracy of the method [30]. Because of this, a relatively fine Eulerian mesh is desirable. Due to the coupling between the temporal and spatial discretizations of the lattice Boltzmann equation this places a stringent constraint on the time step. For LBM-IBM simulations this limitation results in a time step that is orders of magnitude smaller than the elastic and fluid timescales of the problem.

The large separation between the relevant temporal and spatial scales suggests that de-coupling the LBM time step from the physical time step by employing a DTS scheme could reduce the computational time required for capsule simulations. Applications of DTS have been reported for spectral [36] and finite volume [37] models of the lattice Boltzmann equation. More recently a DTS procedure was incorporated into a LBM-IBM scheme for simulation of flow past a cylinder [38]. The transient terms were added as a source into the LBE, and the resulting equations are solved using the multigrid lattice Boltzmann method originally developed by Mavriplis in [29]. The authors reported speedup of approximately 4 when compared with the traditional LBM for unsteady flow past a cylinder [38].

In this work we propose a quasisteady implementation of the LBM-IBM capsule model, treating each time step as a steady flow problem. This allows us to de-couple the LBM time discretization, which is tied to the spatial discretization, from the capsule discretization. This allows for a physical time step orders of magnitude larger than the one required by traditional LBM-IBM capsule models. Neglecting the transient terms of the incompressible Navier-Stokes equations reduces the computational and memory requirements when compared with the method proposed in [38] without sacrificing accuracy in the low Reynolds number regime of capsule deformation. 


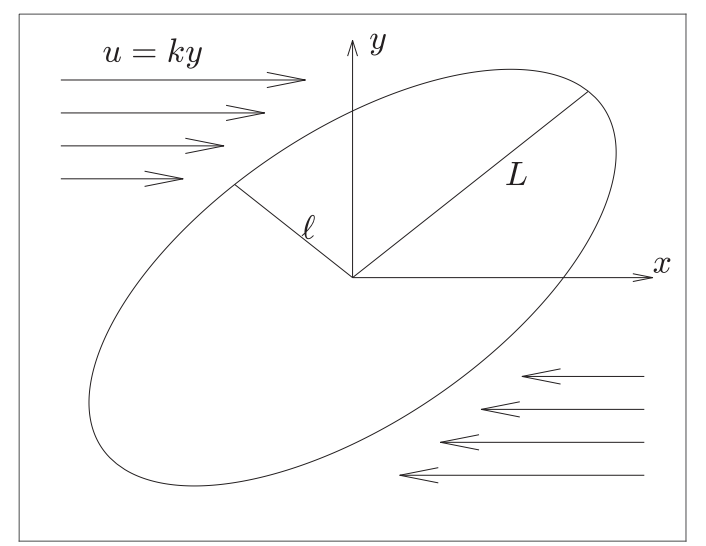
flow.

FIG. 1. The initial configuration for a spheroidal capsule in shear

The proposed quasisteady scheme also allows for easy incorporation into pre-existing LBM-IBM capsule codes, offering efficiency gains of one order of magnitude with the newly proposed scheme.

The structure of this paper is as follows: Sec. II discusses the physical model of capsule deformation in shear flow. Section III discusses the multigrid lattice Boltzmann method. Section IV presents the methods used for computation of the capsule forces. Section V discusses the fluid-structure interaction algorithm. Section VI discusses the coupling of the multigrid LBM to the capsule model. Section VII presents the results from the capsule model for a variety of capsule geometries and parameters. Section VIII discusses the efficiency gains resulting from the multigrid DTS implementation. Section IX concludes the paper with a discussion of the validity and applicability of the quasisteady scheme proposed here.

\section{PROBLEM DESCRIPTION}

In this work we simulate the flow-induced deformation of a capsule in shear flow. The capsule membrane is modeled as a two-dimensional, fluid-filled, elastic membrane surrounded by an ambient fluid. The plane of shear is the $x$-y plane, with periodic boundaries in the $z$ coordinate direction. The capsule is initially inclined by $\pi / 4$ radians with respect to the $x$ axis.

A schematic illustration of the plane of shear can be seen in Fig. 1. The shear rate for the flow is $[k]=\mathrm{s}^{-1}$. The ambient fluid has viscosity, $\left[\mu_{a}\right]=\mathrm{Pas}$, and the interior fluid has viscosity $\left[\mu_{c}\right]=$ Pas. The capsule size is parametrized by the equivalent radius, $[a]=\mathrm{m}$, defined as the radius of a sphere with the same volume as the capsule. The elasticity of the capsule is parametrized by the Young's surface modulus, $\left[E_{s}\right]=\mathrm{Nm}^{-1}$, and the capsule's bending resistance is parametrized by the bending stiffness modulus, $\left[E_{B}\right]=\mathrm{J}$.

These parameters can be combined into a number of dimensionless parameters that characterize the dynamics of shear-induced capsule deformation. The capillary number, also referred to as the dimensionless shear rate by some researchers, is the ratio of the viscous to elastic forces and is given by

$$
\mathrm{Ca}=\frac{\mu_{a} a k}{E_{s}}
$$

The dimensionless bending stiffness is the ratio of the bending forces to the elastic forces and is given by

$$
E_{b}=\frac{E_{B}}{a^{2} E_{s}} .
$$

The viscosity ratio is the ratio of the internal capsule viscosity, $\mu_{c}$, to the ambient fluid viscosity, $\mu_{a}$ :

$$
V=\frac{\mu_{c}}{\mu_{a}} .
$$

For numerical studies typical ranges of these parameters are as follows: $\mathrm{Ca}=0.01-1, V=0.1-10$, and $E_{b}=0-0.1$.

For shear-induced capsule deformation the Reynolds number is given by

$$
\operatorname{Re}=\frac{\rho k a^{2}}{\mu_{a}} .
$$

For biological and microcapsule applications $\mathrm{Re} \ll 1$ $[3,4,39,40]$ and thus, numerical studies often use the Stokes approximation $[12,15,16,41]$. Numerical studies that do consider the fluid inertia use Reynolds numbers in the range of $\operatorname{Re}=0.01-0.1[21,22,26,30,31,42]$.

The capsule dynamics are analyzed by looking at the the deformation parameter, $D_{x y}$, and the inclination angle, $\theta$. Initially proposed by Taylor [43], the deformation parameter of a two dimensional capsule is computed from the lengths of the major and minor axes (denoted by $L$ and $\ell$, respectively, in Fig. 1) of the capsule as follows:

$$
D_{x y}=\frac{L-\ell}{L+\ell} .
$$

The inclination angle is the angle between the capsule's major axis and the $\mathrm{x}$ coordinate axis.

For spherical capsules at moderate capillary numbers $(\mathrm{Ca} \lesssim 0.2)$ the capsule will deform until reaching an approximately constant equilibrium shape [5,6]. After reaching a constant shape, the flow causes the membrane to rotate around the internal fluid without further deformation. This phenomenon, known as tank-treading, has also been observed experimentally for spherical [40] and biconcave [1] capsules.

The dynamics of capsules with nonspherical geometries and capsules at larger values of $\mathrm{Ca}$ exhibit a wide variety of behaviors $[4,8,9,40]$. In general, higher values of $\mathrm{Ca}$ result in more deformation and greater rotation of the capsule toward the $x$ axis. For large values of $\mathrm{Ca}$, capsule breakup will occur [39]. For nonspherical capsules the dynamics typically do not reach a steady deformed shape or orientation, and instead orientation and deformation will exhibit oscillatory or intermittent behavior $[3,8]$.

\section{FLUID SOLVER}

The fluid motion inside and outside the capsule is governed by the incompressible Navier-Stokes equations:

$$
\begin{gathered}
\rho\left(\partial_{t} \mathbf{u}+\mathbf{u} \cdot \nabla \mathbf{u}\right)=-\nabla p+\mu \nabla^{2} \mathbf{u}+\mathbf{p}, \\
\nabla \cdot \mathbf{u}=0,
\end{gathered}
$$

where $\rho$ is the fluid density, $\mathbf{u}$ is the fluid velocity, $\mu$ is the fluid viscosity, $p$ is the pressure, and $\mathbf{p}$ is the external force. 
As discussed above, the typical Reynolds number for numerical simulations of capsule deformation are on the order of $10^{-2}$, and thus the inertial terms in the Navier-Stokes equations have only a small effect on the flow field. Due to this observation, we neglect the transient term in Eq. (6) and instead solve the steady-state system:

$$
\begin{gathered}
\rho \mathbf{u} \cdot \nabla \mathbf{u}=-\nabla p+\mu \nabla^{2} \mathbf{u}+\mathbf{p}, \\
\nabla \cdot \mathbf{u}=0,
\end{gathered}
$$

at each time step. This allows us to de-couple the LBM discretization from the temporal discretization. Furthermore, treating the fluid computation at each time step as a steady flow, we can use the multigrid lattice Boltzmann method proposed in Ref. [29] to accelerate the fluid computation.

\section{A. The lattice Boltzmann method}

In this work we solve Eqs. (8) and (9) using the lattice Boltzmann method (LBM). In LBM the fluid is modeled as a system of discrete particles whose distribution function evolves based on the lattice Boltzmann equation (LBE) given by

$$
f_{i}\left(\mathbf{x}+\mathbf{c}_{i} \delta t, t+\delta t\right)-f_{i}(\mathbf{x}, t)=\Omega_{i}(f), i=0,1, \ldots, N
$$

where $\Omega$ describes the collisions between particles, $h$ is the spatial step, $\delta t$ is the time step, $N+1$ is the number of discrete velocities in the model, $\mathbf{c}_{i}$ is the $i$ th discrete velocity, and $f_{i}$ is the distribution function associated with the $i$ th velocity.

The discretization of space, time, and velocity in the LBE are interrelated as follows:

$$
\delta t=\frac{\Delta x}{c}, \quad \mathbf{c}_{i}=c \mathbf{e}_{i}, \quad c_{s}=\frac{1}{\sqrt{3}} c,
$$

where $c_{s}$ is the speed of sound in the material and $c$ is dependent on the lattice. For the D3Q19 discretization used here, the values of $\mathbf{e}_{i}$ are given as

$$
\begin{aligned}
& {\left[\mathbf{e}_{0}, \mathbf{e}_{1}, \mathbf{e}_{2}, \ldots, \mathbf{e}_{18}\right]} \\
& =\left(\begin{array}{ccccccccccccccccccc}
0 & 1 & -1 & 0 & 0 & 0 & 0 & 1 & 1 & -1 & -1 & 1 & -1 & 1 & -1 & 0 & 0 & 0 & 0 \\
0 & 0 & 0 & 1 & -1 & 0 & 0 & 1 & -1 & 1 & -1 & 0 & 0 & 0 & 0 & 1 & 1 & -1 & -1 \\
0 & 0 & 0 & 0 & 0 & 1 & -1 & 0 & 0 & 0 & 0 & 1 & 1 & -1 & -1 & 1 & -1 & 1 & -1
\end{array}\right) .
\end{aligned}
$$

For the above $\mathbf{e}_{\mathbf{i}}$, a choice of $c=1 \mathrm{~m} / \mathrm{s}$ results in the particle distribution functions moving exactly one grid point at each time step, eliminating the need for interpolation.

In this work, the collision term, $\Omega$, in Eq. (10) is approximated using the multiple relaxation time (MRT) collision model discussed in Ref. [44]. The MRT collision operator maps the distribution function to the physical moments of the system where each moment is relaxed to its corresponding equilibrium moment. With the above discretization, the MRT collision model can be implemented in the following two-step procedure:

$$
\begin{aligned}
& \text { Collision: } f_{i}^{*}(\mathbf{x})=f_{i}\left(\mathbf{x}, t_{n}\right)-M_{i j}^{-1} S_{j k}\left[M_{k l} f_{l}\left(\mathbf{x}, t_{n}\right)-m_{k}^{e q}\left(\mathbf{x}, t_{n}\right)\right], \\
& \text { Advection: } f_{i}\left(\mathbf{x}+\mathbf{c}_{i} \delta t, t_{n}+\delta t\right)=f_{i}^{*}(\mathbf{x}), \quad i=0,1, \ldots, 18 .
\end{aligned}
$$

Here, $m_{k}^{\mathrm{eq}}$ is the $k$ th equilibrium moment, $S_{j k}$ are the components of the relaxation matrix, $\mathbf{S}$, and $M_{j k}$ are the components of the collision matrix mapping $f$ to the moment space. $\mathbf{m}^{\mathrm{eq}}$ and the collision matrix, $\mathbf{M}$, are the same as derived in Ref. [44].

The relaxation matrix, $\mathbf{S}$, is defined as

$$
\begin{aligned}
\mathbf{S}= & \operatorname{diag}\left(s_{0}, s_{1}, s_{2}, s_{3}, s_{4}, s_{5}, s_{6}, s_{7}, s_{8}, s_{9}, s_{10},\right. \\
& \left.s_{11}, s_{12}, s_{13}, s_{14}, s_{15}, s_{16}, s_{17}, s_{18}\right) .
\end{aligned}
$$

$s_{0}, s_{3}, s_{5}$, and $s_{7}$ correspond to the conserved moments and hence are set equal to zero. The parameters $s_{4}=s_{6}=$ $s_{8}=s_{9}=s_{11}=s_{13}=s_{14}=s_{15}$ are related to the kinematic viscosity, $v$, as follows:

$$
v=c_{s}^{2} \delta t\left(\frac{1}{s_{4}}-\frac{1}{2}\right) .
$$

$s_{1}$ is related to the bulk viscosity, $\xi$, by

$$
\xi=\frac{2}{3} c_{s}^{2} \delta t\left(\frac{1}{s_{1}}-\frac{1}{2}\right)
$$

The parameters, $s_{2}, s_{10}, s_{12}, s_{16}, s_{17}, s_{18}$ correspond to higher order moments that do not affect the solutions in the hydrodynamic regime and are thus set to 1.8 [44].

Once the distribution function has been updated we can compute the macroscopic variables associated with Eqs. (8) and (9) by computing moments of the distribution function as follows:

$$
\rho=\sum_{i=0}^{N} f_{i}, \quad \mathbf{j}=\rho \mathbf{u}=\sum_{i=0}^{N} f_{i} \mathbf{c}_{i} .
$$

Additional macroscopic variables can be obtained by computing higher order moments of the distribution function and are defined by the rows of matrix $\mathbf{M}$ [44].

To incorporate the forces, $\mathbf{p}(\mathbf{x})$, into the LBM we follow the method described in Ref. [45]. First, the momentum computed using Eq. (17) is updated as follows:

$$
\mathbf{j}^{\prime}(\mathbf{x})=\mathbf{j}(\mathbf{x})+\frac{\delta t}{2} \mathbf{p}(\mathbf{x}) .
$$

Next, $\mathbf{j}^{\prime}$ is used to compute the equilibrium moments, $\mathbf{m}^{e q}$, in Eq. (13). After computing the equilibrium moments, the 
momentum is updated once more using

$$
\mathbf{j}^{\prime \prime}(\mathbf{x})=\mathbf{j}^{\prime}(\mathbf{x})+\frac{\delta t}{2} \mathbf{p}(\mathbf{x})
$$

Finally, $\mathbf{j}^{\prime \prime}$ is used to update the moments corresponding to the fluid momentum, $m_{3}, m_{5}$, and $m_{7}$.

\section{B. The multigrid lattice Boltzmann method}

For steady flows the LBM becomes an iterative procedure. Due to the improved error damping properties of relaxation schemes, Mavriplis [29] introduced a relaxation step following the collision and advection stages of the LBM, resulting in an iterative method analogous to the Jacobi under-relaxation scheme for linear systems. On the fine grid the Jacobi underrelaxation scheme is carried out in the following three-step procedure:

$$
\begin{aligned}
& \text { Collision: } f_{i}^{*}(\mathbf{x})=f_{i}^{n}(\mathbf{x})-M_{i j}^{-1} S_{j k}\left[M_{k l} f_{l}(\mathbf{x})-m_{k}^{\mathrm{eq}}(\mathbf{x})\right], \\
& \text { Advection: } f_{i}^{* *}\left(\mathbf{x}+\mathbf{e}_{i} \Delta x\right)=f_{i}^{*}(\mathbf{x}), i=0,1, \ldots, 18, \\
& \text { Relaxation: } f_{i}^{n+1}(\mathbf{x})=\gamma f_{i}^{* *}(\mathbf{x})+(1-\gamma) f_{i}^{n}(\mathbf{x}),
\end{aligned}
$$

where $\gamma$ is a relaxation parameter such that $0<\gamma<1$.

A key component of multigrid schemes is the residual, which is used to estimate the error of the iterative solution. For the LBE the $i$ th component of the residual is given by

$$
\begin{aligned}
r_{i}(\mathbf{f})= & f_{i}(\mathbf{x})-f_{i}\left(\mathbf{x}-\mathbf{e}_{i} \Delta x\right)+M_{i j}^{-1} S_{j k} \\
& \times\left[M_{k l} f_{l}\left(\mathbf{x}-\mathbf{e}_{i} \Delta x\right)-m_{k}^{e q}\left(\mathbf{x}-\mathbf{e}_{i} \Delta x\right)\right],
\end{aligned}
$$

where $\mathbf{f}:=\left[f_{0}, f_{2}, \ldots, f_{18}\right]^{T}$. On the fine grid the error equation is given by

$$
r_{i}^{h}\left(\mathbf{f}_{h}\right)=0
$$

After solving this system for $\mathbf{f}_{h}$, we approximate the error by computing the residual using Eq. (21). We then use pointwise injection to map the distribution function to the coarse grid and an averaging operator to map the error to the coarse grid.

On the coarse grid levels, the correction equation is written as

$$
\begin{gathered}
r_{i}^{H}\left(\mathbf{f}_{H}\right)=D_{i}^{H}, \\
D_{i}^{H}=r_{i}^{H}\left(\hat{I}_{h}^{H} \mathbf{f}_{h}\right)-2 I_{h}^{H} r_{i}^{h}\left(\mathbf{f}_{h}\right),
\end{gathered}
$$

where $r_{i}^{H}$ is computed using Eq. (21) and $D_{i}^{H}$ is known as the defect correction. The factor of 2 in the second term on the right side of Eq. (24) is used to scale the relaxation parameters on the coarse grids [29]. Equation (23) is solved for $\mathbf{f}_{H}$ in almost the same manner as Eq. (20), with a modification to the relaxation step so that $D_{i}^{H}$ can be added to the distribution function as follows:

Relaxation: $f_{i}^{n+1}(\mathbf{x})=\gamma\left[f_{i}^{* *}(\mathbf{x})+D_{i}^{H}(\mathbf{x})\right]+(1-\gamma) f_{i}^{n}(\mathbf{x})$.

After solving for the error on the coarse grid, the error is mapped back to the fine grid using an averaging operator and is added to our approximation of the distribution function.

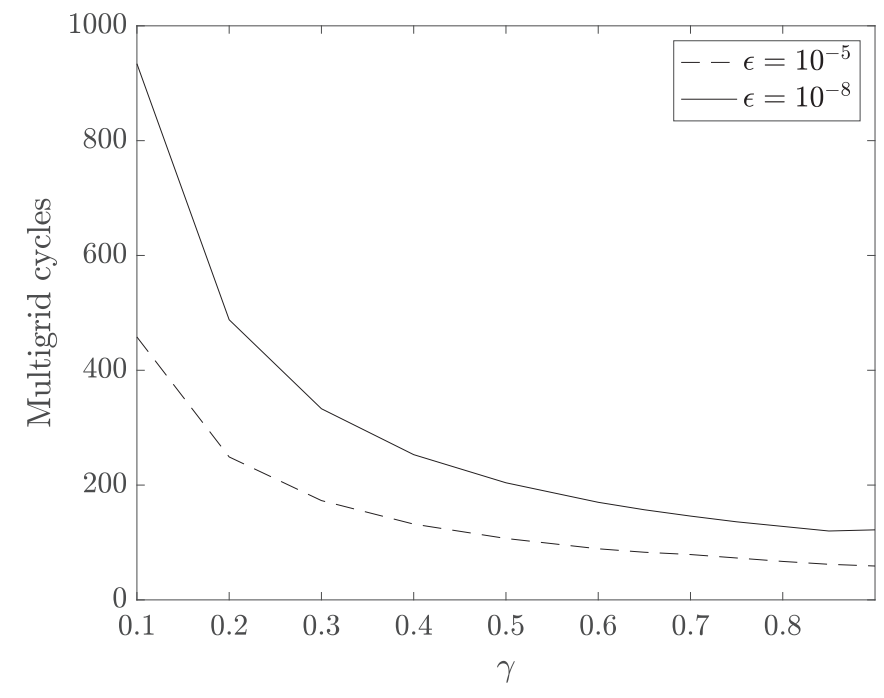

FIG. 2. The number of multigrid iterations for solution of liddriven cavity flow using the multigrid lattice Boltzmann method for various values of the relaxation parameter, $\gamma$.

\section{Selecting the relaxation parameter}

To determine the optimal value of $\gamma$ in Eq. (20) we conduct an experiment similar to the two-dimensional study conducted by Patil et al. [46]. Simulations are conducted for threedimensional lid driven cavity flow with the primary Reynolds number used in this paper, $\operatorname{Re}=0.05$. In this work the fluid velocity is assumed to have converged to the correct velocity field when the following constraint has been met:

$$
\frac{\sum_{i}\left|\mathbf{u}^{n+1}\left(\mathbf{x}_{i}\right)-\mathbf{u}^{n}\left(\mathbf{x}_{i}\right)\right|_{2}}{\sum_{i}\left|\mathbf{u}^{n+1}\left(\mathbf{x}_{i}\right)\right|_{2}} \leqslant \epsilon .
$$

In Eq. (26) $\epsilon$ is a user-defined tolerance and $|\cdot|_{2}$ is the $L^{2}$ norm.

For each simulation the number of multigrid cycles needed to reach a tolerance of $\epsilon=10^{-5}$ and $\epsilon=10^{-8}$ is recorded. The domain of the simulations is $[0,1]^{3}$, with a discretization of $N=129$ nodes in each direction. The $w$-cycle multigrid schedule was used for these tests.

In Fig. 2 the number of multigrid cycles required to reach convergence for values of $\gamma$ ranging from 0.1 to 0.9 is shown. The trend for both values of $\epsilon$ is the same: increasing $\gamma$ results in faster convergence until reaching a minimum between 0.8 and 0.9 . Based on this, we have set $\gamma=0.8$ for the simulations presented below.

\section{CAPSULE MODEL}

The capsule membrane is modeled as a two-dimensional surface with transverse forces caused by in-plane tensions and normal forces caused by the capsule's bending resistance. The membrane discretization is a triangular mesh where each triangle's vertices are tracked as Lagrangian nodes. The positions of the vertices are advected based on the fluid velocity near the capsule membrane using the immersed boundary method [13]. In this work we consider three capsule geometries: spherical, spheroidal and biconcave discoid. The mesh 


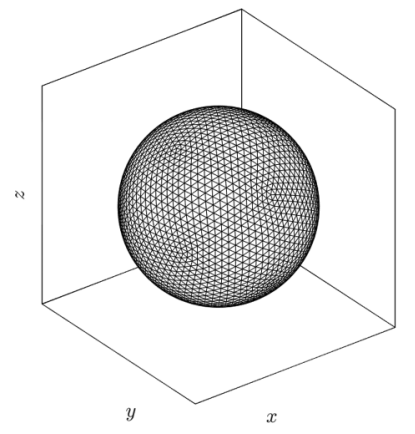

(a)

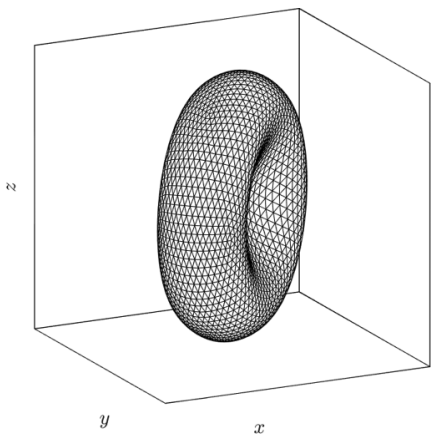

(b)
FIG. 3. (a) The spherical and (b) biconcave meshes used in this work. These discretizations have 5120 triangles composed of 2562 Lagrangian nodes.

used for the spherical and biconcave capsules can be seen in Figs. 3(a) and 3(b), respectively.

\section{A. Elastic forces}

The elasticity of the capsule is modeled by a constitutive law describing the strain energy of the membrane. In this work we use two strain energy functions: the neo-Hookean law and Skalak's law. The neo-Hookean law was originally developed for three-dimensional solids, and is a function of the three dimensional principle stretches, $\lambda_{1}, \lambda_{2}$, and $\lambda_{3}$. By assuming volume incompressibility, $\lambda_{1} \lambda_{2} \lambda_{3}=1$, we can write the strain energy function in terms of $\lambda_{1}$ and $\lambda_{2}$ in the following form:

$$
W^{N H}=\frac{E_{s}}{6}\left[\lambda_{1}^{2}+\lambda_{2}^{2}+\lambda_{1}^{-2} \lambda_{2}^{-2}-3\right] .
$$

The second strain energy function used in this work is Skalak's law. Proposed by Skalak et al. [47], Skalak's law is a two-dimensional constitutive law developed to account for the resistance to surface area dilation exhibited by red blood cell membranes. The strain energy function for Skalak's law is given by

$$
\begin{aligned}
W^{S K}= & \frac{E_{s}}{8}\left[\left(\lambda_{1}^{2}+\lambda_{2}^{2}-2\right)^{2}+2\left(\lambda_{1}^{2}+\lambda_{2}^{2}-2\right)\right. \\
& \left.-2\left(\lambda_{1}^{2} \lambda_{2}^{2}-1\right)+C\left(\lambda_{1}^{2} \lambda_{2}^{2}-1\right)^{2}\right] .
\end{aligned}
$$

The final term on the right-hand side of Eq. (28) can be used to approximate area incompressibility by selecting $C \gg 1$. Although Skalak's law was designed to capture the areaincompressible red blood cell membrane, for values of $C \sim$ $\mathcal{O}(1)$ the law can be used to simulate a general elastic membrane $[41,48]$.

It should be noted that there are multiple definitions of the surface elasticity modulus in the literature, varying by a constant scalar. For the neo-Hookean law we follow the definition used in Refs. [12,22], which results in $E_{s}=3 G_{\mathrm{nh}}$, where $G_{\mathrm{nh}}$ is the neo-Hookean elasticity modulus used in Ref. [48]. For Skalak's Law we follow the definition used in Refs. [22,26], which results in $E_{s}=2 G_{\text {sk }}$, where $G_{\text {sk }}$ is the Skalak elasticity modulus used in [48]. Care should be taken when comparing results from different researchers as the capillary number, $\mathrm{Ca}$, Skalak parameter, $C$, and dimensionless bending modulus, $E_{b}$,

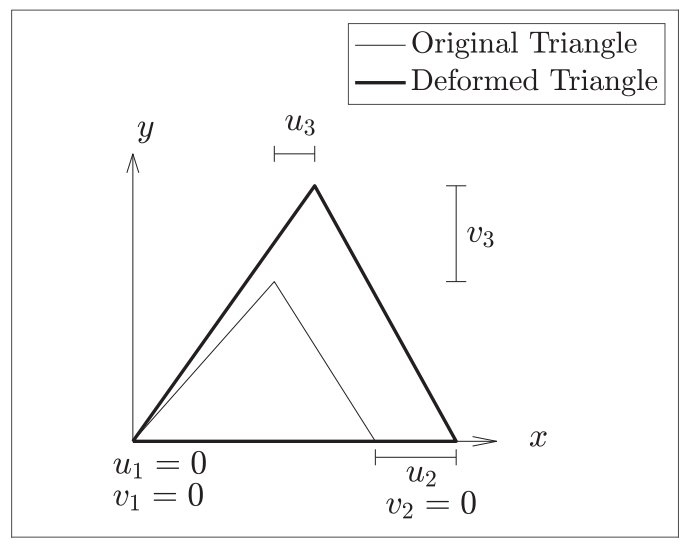

FIG. 4. Computation of the deformation is done by mapping both triangles to a common plane.

all depend on the definition of the surface elasticity modulus that is used.

With a constitutive law chosen, the capsule's elastic forces are computed using the linear finite element method originally proposed by Charrier et al. [32]. First, as shown in Fig. 4, each deformed triangular element is mapped into a common plane with its initial configuration, whereby the displacement vector, $[u, v]^{T}$, can be easily computed at each vertex of the triangle. The displacement vector is then fitted to a linear shape function, allowing for the computation of the right Cauchy-Green tensor and its eigenvalues, the principle stretch ratios, $\lambda_{1}$ and $\lambda_{2}$.

With $u^{k}, v^{k}, \lambda_{1}$, and $\lambda_{2}$ known we can use the principle of virtual work to obtain the following relation for the in-plane forces at the k-th vertex of the triangle:

$$
\begin{aligned}
P_{x}^{k} & =V_{e} \frac{\partial W}{\partial \lambda_{1}} \frac{\partial \lambda_{1}}{\partial u^{k}}+V_{e} \frac{\partial W}{\partial \lambda_{2}} \frac{\partial \lambda_{2}}{\partial u^{k}} \\
P_{y}^{k} & =V_{e} \frac{\partial W}{\partial \lambda_{1}} \frac{\partial \lambda_{1}}{\partial v^{k}}+V_{e} \frac{\partial W}{\partial \lambda_{2}} \frac{\partial \lambda_{2}}{\partial v^{k}} .
\end{aligned}
$$

Here $W$ is the strain energy function given by either Eq. (27) or Eq. (28) and $V_{e}$ is the element area in the undeformed state. The forces at each vertex are then mapped back to the physical coordinates and added to the node's Lagrangian force term. For a mesh with 5120 triangles, each node is the vertex of five or six triangles. The full elastic force at a given Lagrangian node, $\mathbf{P}_{\mathbf{e}}$, is the sum of the forces at each of these five or six triangles.

\section{B. Bending forces}

The capsule's bending resistance is modeled using the shape energy functional proposed by Helfrich in Ref. [33]:

$$
W_{b}=\frac{E_{B}}{2} \int_{S}\left(2 \kappa-c_{0}\right)^{2} d S,
$$

where $\kappa$ is the mean curvature, $c_{0}$ is the spontaneous curvature, and $E_{B}$ is the bending stiffness modulus of the capsule. The spontaneous curvature is a parameter that defines the reference configuration for the capsule's bending energy. 
The bending force density, $\mathbf{f}_{\mathbf{b}}$, is obtained by taking the first variation of Eq. (30), which we can express in terms of the mean curvature, $\kappa$, spontaneous curvature, $c_{0}$, and Gaussian curvature, $\kappa_{g}$ as follows [34]:

$$
\mathbf{f}_{\mathbf{b}}=E_{B}\left[\left(2 \kappa+c_{0}\right)\left(2 \kappa^{2}-2 \kappa_{g}-c_{0} \kappa\right)+2 \Delta_{\mathrm{LB}} \kappa\right] \mathbf{n},
$$

where $\Delta_{\mathrm{LB}}$ is the Laplace-Beltrami operator. The nodal bending force, $\mathbf{P}_{\mathbf{b}}$, is obtained from Eq. (31) by multiplying $\mathbf{f}_{\mathbf{b}}$ by a given node's Voroni area, $A_{\mathrm{vor}}$ [49].

Computation of the various terms in Eq. (31) has been the subject of reviews by Guckenberg et al. [49,50]. In this work we compute the curvatures in Eq. (31) using the method proposed by Garimella and Swartz [51], where, at each node, a quadratic surface is fitted to the node and its nearest neighbors. The mean and Gaussian curvatures, as well as the normal vector, of the Lagrangian nodes, can then be approximated from their analytical expressions using the standard formulas for an implicit surface [51].

The only term in Eq. (31) remaining to be calculated is the Laplace-Beltrami of the mean curvature, $\Delta_{\mathrm{LB}} \kappa$. Here we use the cotan Laplacian outlined in the review by Reuter et al. [52]:

$\Delta_{\mathrm{LB}} \kappa\left(\mathbf{X}_{i}\right)=\frac{1}{2 A_{\mathrm{vor}}} \sum_{j \in N\left(\mathbf{X}_{i}\right)}\left(\cot \alpha_{i j}+\cot \beta_{i j}\right)\left[\kappa\left(\mathbf{X}_{i}\right)-\kappa\left(\mathbf{X}_{j}\right)\right]$,

where $\alpha_{i j}$ and $\beta_{i j}$ are the exterior angles of the two triangles containing the edge created by $X_{j}$ and $X_{i}$ [53]. Consistent with the notation above, $A_{\text {vor }}$ is the area of the Voroni region.

\section{FLUID-STRUCTURE INTERACTION}

\section{A. Immersed boundary method}

The interaction between the membrane and surrounding fluid is computed using the immersed boundary method (IBM). Developed by Peskin and reviewed in Ref. [13], in IBM the capsule is modeled as a set of Lagrangian coordinates suspended in a fluid-filled domain described using Eulerian coordinates. The computation of the Lagrangian fluid velocity, $\mathbf{U}$, from the Eulerian flow velocity, $\mathbf{u}$, and the Eulerian external force, $\mathbf{p}$, from the Lagrangian forces, $\mathbf{P}$, are achieved through the following integrals:

$$
\begin{aligned}
& \mathbf{U}\left(\mathbf{X}_{c}\right)=\int_{V} \mathbf{u}\left(\mathbf{x}_{j}\right) \delta\left(\mathbf{X}_{c}-\mathbf{x}_{j}\right) d \mathbf{x}, \\
& \mathbf{p}\left(\mathbf{x}_{j}\right)=\int_{S} \mathbf{P}\left(\mathbf{X}_{c}\right) \delta\left(\mathbf{X}_{c}-\mathbf{x}_{j}\right) d \mathbf{X} .
\end{aligned}
$$

The Lagrangian force, $\mathbf{P}$, in Eq. (34) is given by $\mathbf{P}=-\mathbf{P}_{\mathbf{b}}-$ $\mathbf{P}_{\mathbf{e}}$, where $\mathbf{P}_{\mathbf{b}}$ and $\mathbf{P}_{\mathbf{e}}$ are the bending and elastic forces at a given node. The negative signs reflect the fact that the forces are exerted by the capsule on the fluid [49].

In this work, the integrals in Eqs. (33) and (34) are approximated numerically using the following discrete delta function:

$$
\delta_{h}(x)= \begin{cases}\frac{1}{4 \Delta x}\left[1+\cos \frac{\pi x}{2 \Delta x}\right] & |x| \leqslant 2 \Delta x \\ 0 & |x|>2 \Delta x .\end{cases}
$$

With this approximation and the convention that $\delta_{h}(\mathbf{X})=$ $\delta_{h}(x) \delta_{h}(y) \delta_{h}(z)$, the computation of the integrals in Eqs. (33) and (34) can be written as

$$
\begin{aligned}
\mathbf{U}\left(\mathbf{X}_{c}\right) & =\sum \mathbf{u}\left(\mathbf{x}_{j}\right) \delta_{h}\left(\mathbf{X}_{c}-\mathbf{x}_{j}\right) \Delta x^{3}, \\
\mathbf{p}\left(\mathbf{x}_{j}\right) & =\sum \mathbf{P}\left(\mathbf{X}_{c}\right) \delta_{h}\left(\mathbf{X}_{c}-\mathbf{x}_{j}\right) \Delta x^{2} .
\end{aligned}
$$

The forces, $\mathbf{p}$, computed above are then incorporated into the LBM model through Eqs. (18) and (19), which give us the moments of the distribution function used to compute the Eulerian fluid velocity as described above in Sec. III.

Once the Lagrangian velocity has been obtained we can update the capsule's position through numerical integration of the following equation:

$$
\frac{\partial \mathbf{X}_{c}}{\partial t}=\mathbf{U}\left(\mathbf{X}_{c}, t\right) .
$$

In this work we use second-order Adams-Bashforth to integrate Eq. (37), giving us the following formula for updating the capsule nodes:

$$
\mathbf{X}_{c}\left(t_{n+1}\right)=\mathbf{X}_{c}\left(t_{n}\right)+\frac{\Delta t}{2}\left[3 \mathbf{U}\left(\mathbf{X}_{c}, t_{n+1}\right)-\mathbf{U}\left(\mathbf{X}_{c}, t_{n}\right)\right] .
$$

Note that in our multigrid implementation, $\Delta t$ is not the same value as $\delta t$, the time step in the lattice Boltzmann equation. In fact, as discussed below, values of $\Delta t$ several orders of magnitude larger than $\delta t$ are sufficient to produce physically accurate results using the multigrid implementation outlined in Sec. VI.

For explicit numerical integration of the capsule position, such as the scheme proposed here, the stability of the algorithm is given as follows [54-56]:

$$
\Delta t<\alpha \frac{\mathrm{Ca} h}{k a},
$$

where $h$ is the size of the mesh elements and $\alpha$ is a constant that depends on the method of integration used to solve Eq. (37).

\section{B. Fluid viscosity}

When there is a jump between the viscosity inside the capsule, $\mu_{c}$, and the viscosity of the ambient fluid, $\mu_{a}$, we use the front-tracking method originally proposed by Zhang $\mathrm{et} \mathrm{al}$. [35] for LBM-IBM models. A smoothed heaviside function is used to compute the viscosity at points near the capsule membrane as follows:

$$
\mu(\mathbf{x})=\mu_{c}+\left(\mu_{a}-\mu_{c}\right) H[d(\mathbf{x})],
$$

where $d(\mathbf{x})$ is the minimum distance between a given Eulerian point, $\mathbf{x}$, and the capsule membrane.

As in Ref. [35], the following continuous approximation to the heaviside function in Eq. (40) is used in this work:

$$
H(d)= \begin{cases}0 & d<-2 \Delta x \\ \frac{1}{2}\left(1+\frac{d}{2 \Delta x}+\frac{1}{\pi} \sin \frac{\pi d}{2 \Delta x}\right) & -2 \Delta x \leqslant d \leqslant 2 \Delta x \\ 1 & d>2 \Delta x\end{cases}
$$

With the viscosity updated we can use $\mu(\mathbf{x})$ in Eq. (15) to update the corresponding relaxation parameters associated with the LBM. 


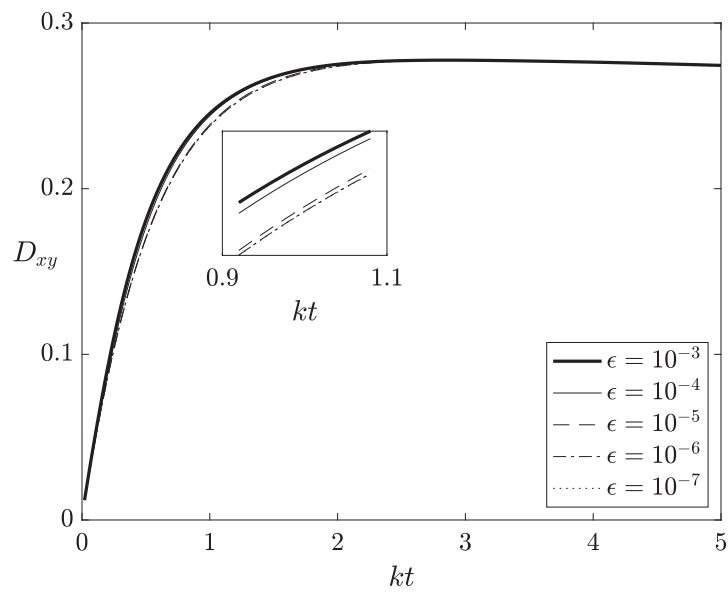

(a)

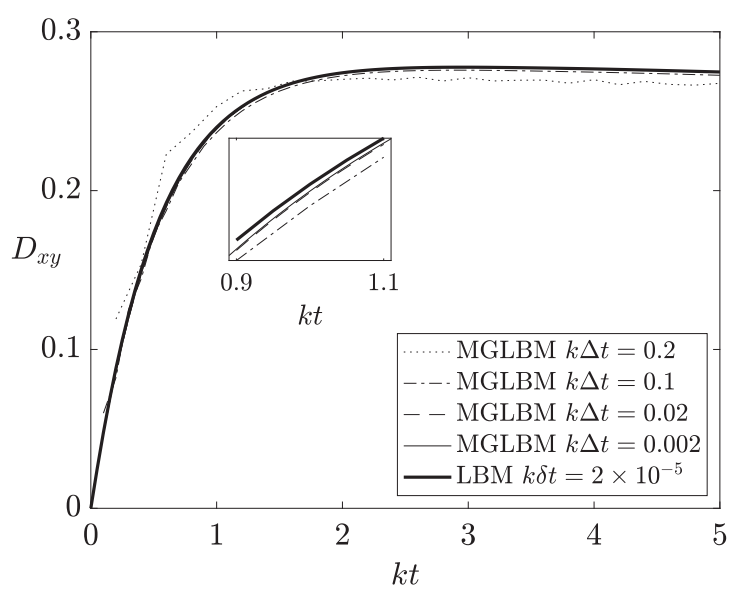

(b)

FIG. 5. (a) $D_{x y}$ for various choices of the iterative tolerance, $\epsilon$. (b) $D_{x y}$ for various time steps, $\Delta t$.

\section{MULTIGRID IMPLEMENTATION}

To demonstrate the potential for speedup offered by a DTS scheme, we first estimate the physical timescales of the problem using $k, \mu_{a}, E_{s}, E_{b}$, and $a$. The fluid timescale is given by $\tau_{f}=k^{-1}$, the elastic timescale is given by $\tau_{e}=\mu_{a} a / E_{s}=$ $\mathrm{Ca} k^{-1}$ and, when bending stiffness is considered, the bending modulus timescale is given by $\tau_{b}=\mu_{a} a /\left(E_{b} E_{s}\right)=\mathrm{Ca} k^{-1} / E_{b}$. Since typical parameter ranges are given by $\mathrm{Ca} \sim 10^{-2}-1$ and $E_{b} \sim 10^{-3}-10^{-1}, \tau_{e}$ is the most restrictive timescale.

To illustrate the limitations of the standard LBM-IBM time step we consider the ratio of $\tau_{e}$ to the LBM time step:

$$
\frac{\tau_{e}}{\delta t}=\frac{\mathrm{Ca}}{k \delta t} \text {. }
$$

In the LBM-IBM capsule model, $\delta t$ is determined by the grid resolution based on Eq. (11), and thus, for a fixed value of $\mathrm{Ca}$ and a fixed grid resolution, $\tau_{e} / \delta t$ can only be adjusted by the shear rate, $k$. The shear rate is restricted by the stability constraint given by Eq. (39). In this work we find that a shear rate, $k$, on the order of $10^{-4} \mathrm{~s}^{-1}$ is required for numerical stability. Inserting $k=10^{-4} \mathrm{~s}^{-1}$ and the time step determined by the Eulerian grid resolution, $\delta t=0.1 \mathrm{~s}$, the ratio into Eq. (42) results in $\tau_{e} / \delta t \sim 10^{5} \mathrm{Ca}$. The large value of $\tau_{e} / \delta t$ indicates that there is a large separation between the physical and LBM timescales of the problem.

To de-couple the physical time step from the LBM algorithm we propose a quasisteady, DTS LBM scheme. At each physical time step, computation of the fluid velocity is treated as a steady-state problem, which is solved iteratively using the multigrid lattice Boltzmann method [29,46,57]. The LBM time step, $\delta t$, defined in Eq. (11) becomes a pseudo-time step related to the iterative LB scheme. This allows us to introduce a new physical time step, $\Delta t$, used to update the capsule position in Eq. (38), which can be adjusted without compromising the lattice structure of the LBM discretization.

\section{A. Convergence criterion}

For iterative solution of the fluid velocity a proper choice of $\epsilon$ in Eq. (26) is essential to obtaining the correct flow. In Fig. 5(a) the deformation parameter, $D_{x y}$, is shown for values of $\epsilon$ ranging from $10^{-3}$ to $10^{-7}$ for simulations of an initially spherical capsule with $\mathrm{Ca}=0.05, V=1, E_{b}=0$, $k=2 \times 10^{-4} \mathrm{~s}^{-1}$, and $k \Delta t=0.02$. As can be seen in the figure, the deformation converges to the correct final deformation for all of the values shown, however, the intermediate dynamics show slight deviations for larger tolerances, $\epsilon$. For $\epsilon=10^{-3}$ and $\epsilon=10^{-4}$ the deformation occurs at a slightly faster pace, however, the curves for $\epsilon \leqslant 10^{-5}$ are all nearly identical.

This is best seen in the inset of Fig. 5(a), which shows the differences between the values of $D_{x y}$ between $k t=0.9$ and $k t=1.1$. The curves for $\epsilon=10^{-5}, \epsilon=10^{-6}$ and $\epsilon=10^{-7}$ exhibit close agreement. The curves for $\epsilon=10^{-3}$ and $\epsilon=$ $10^{-4}$ deviate from the curves for smaller values of $\epsilon$, with $\epsilon=10^{-4}$ showing better agreement than $\epsilon=10^{-3}$, indicating convergence of $D_{x y}$ as $\epsilon \rightarrow 0$. Similar results were obtained for other values of $V, E_{b}, \mathrm{Ca}$ and $\Delta t$ as well as for other initial capsule geometries.

\section{B. Time-step selection}

Because $\Delta t$ is no longer linked to the spatial discretization, selection of the time step can be determined based on the desired accuracy of the simulation. In Fig. 5(b) the deformation parameter is plotted for various values of the dimensionless time step, $k \Delta t$, along with the deformation parameter from the standard MRT LBM for simulations of an initially spherical capsule with $\mathrm{Ca}=0.05, V=1, E_{b}=0$, and $k=2 \times 10^{-4} \mathrm{~s}^{-1}$. For the multigrid LBM simulations a tolerance of $\epsilon=10^{-5}$ was used.

From the figure it is clear that while $k \Delta t=0.2$ captures the qualitative behavior of the deformation, the time step is too large to accurately resolve the dynamics. $k \Delta t=0.1$, $k \Delta t=0.02$, and $k \Delta t=0.002$, however, all closely follow the deformation curve of the standard LBM simulation, with convergence to the standard LBM method as $k \Delta t \rightarrow k \delta t=$ $2 \times 10^{-5}$. From the inset to Fig. 5(b) it can be seen that the curves for $k \Delta t=0.002$ and $k \Delta t=0.02$ fall on top of each other, with only a slight deviation from the deformation obtained from the standard LBM model, while the curve for $k \Delta t=0.1$ shows slightly larger deviations from the standard 


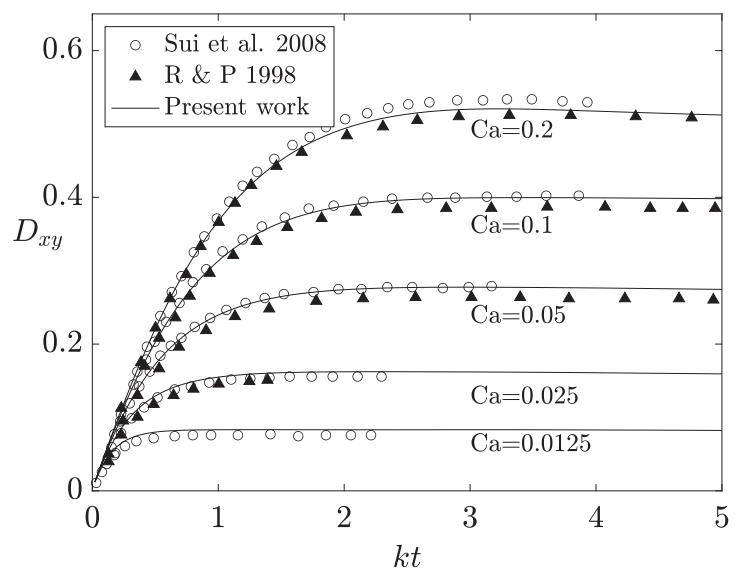

(a)

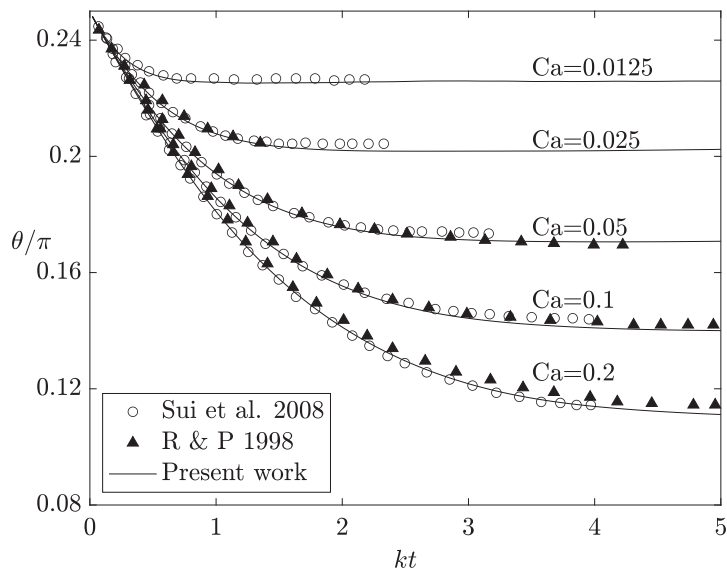

(b)

FIG. 6. (a) The deformation parameter and (b) inclination angle for various values of Ca with $V=1$ and $E_{b}=0$.

LBM curve. Similar results are also found for larger values of Ca.

For a fixed value of $k$, the elastic timescale, $\tau_{e}=\mathrm{Ca} k^{-1}$, is proportional to $\mathrm{Ca}$. To account for this we set $k \Delta t=0.02$ for $\mathrm{Ca} \geqslant 0.05$, while for $\mathrm{Ca}<0.05$ the time step is decreased proportionally with the capillary number. This can be summarized by the following equation:

$$
k \Delta t=\min (0.4 \mathrm{Ca}, 0.02) .
$$

In our simulations Eq. (43) has been found to be sufficient for stable simulations for other values of $k, E_{b}, V$, and $\mathrm{Ca}$, as well as for other capsule geometries.

\section{CAPSULE SIMULATIONS}

\section{A. Numerical setup}

For the simulations presented here a mesh consisting of 2562 Lagrangian nodes, comprising 5120 triangular elements was used. The capsule is placed in the center of the fluid domain with its major axis aligned $\pi / 4$ radians above the $\mathrm{x}$-axis. The Eulerian grid is discretized into a $129^{3}$ grid, with a domain of $[0,12.8 a]$ in $x, y$ and $z$, which results in a step size of $\Delta x=\Delta y=\Delta z=0.1 a$. The simulations below are conducted with a Reynolds number of $\operatorname{Re}=0.05$, a capsule radius of $a=1 \mathrm{~m}$, a density of $\rho=1 \mathrm{~kg} \mathrm{~m}^{-3}$, and a shear rate of $k=2 \times 10^{-4} \mathrm{~s}^{-1}$.

The simulations presented here were conducted with the time step, $\Delta t$, selected using Eq. (43). The convergence criterion for the multigrid LBM was set to $\epsilon=10^{-5}$ based on the results from Sec. VI. Each multigrid cycle uses a fivegrid level $w$-cycle multigrid schedule. The finest grid has a resolution of $129^{3}$ and the coarsest grid has a resolution of $5^{3}$.

The MRT relaxation parameters $s_{4}$ and $s_{1}$ are set according to Eqs. (15) and (16), respectively. The parameters $s_{0}, s_{3}, s_{5}$, and $s_{7}$ correspond to the conserved moments and are thus set equal to zero. The remaining relaxation parameters are set equal to 1.8 .

The deformation parameter and inclination angle are computed using the method discussed in Refs. [12,21]. First, we compute the capsule's moment of inertia:

$$
I_{i j}=\int_{V}\left(r_{k} r_{k} \delta_{i j}-r_{i} r_{j}\right) d V,
$$

where $\mathbf{r}$ is the radial vector, pointing outward from the capsule center. Both $D_{x y}$ and $\theta$ are then obtained from the eigenvalues and eigenvectors of $I$. The eigenvalues of $I$ correspond to the lengths of the axis of an ellipsoid with moment of inertia $I$. The lengths of the major and minor axis for this ellipsoid are used to approximate $L$ and $\ell$ in Eq. (5) [21]. The inclination angle, $\theta$, is computed by finding the principle value of the angle between the capsule's major axis and the $x$ axis.

\section{B. Spherical capsules}

The dynamics of spherical capsules in shear flow have been studied theoretically [5,6] and numerically (e.g., Refs. $[12,14,21,22,30])$ and are well-known. Initially, the flow results in deformation of the sphere until the capsule reaches an ellipsoidal equilibrium shape. After reaching an equilibrium shape, the ambient shear flow causes the membrane to rotate about the internal fluid. This phenomenon, known as tank-treading, has also been observed experimentally for spherical [40] and biconcave [1] capsules.

In Fig. 6 the results for simulations of initially spherical capsules are given for a variety of capillary numbers, Ca. Figures 6(a) and 6(b) plot the deformation parameter and inclination angle of the capsule as a function of time. The results are compared to the published results in Refs. [12,22] showing close agreement. As can be seen in the figures, the flow causes the sphere to gradually deform and rotate toward the $x$ axis. This process occurs until the capsule obtains a steady shape and inclination, as can be seen by the constant values of $D_{x y}$ and $\theta / \pi$ for later values of the simulation. Increased values of $\mathrm{Ca}$ result in greater deformation and more rotation.

Figure 7(a) shows the final shapes obtained from our simulations as a function of the capillary number. As can be seen the final shapes move farther away from a sphere as $\mathrm{Ca}$ is increased. In Fig. 7(b) the trajectory of a particle on the membrane surface is tracked for a capillary number of $\mathrm{Ca}=$ 0.05 . As can be seen, once the capsule reaches its final shape 


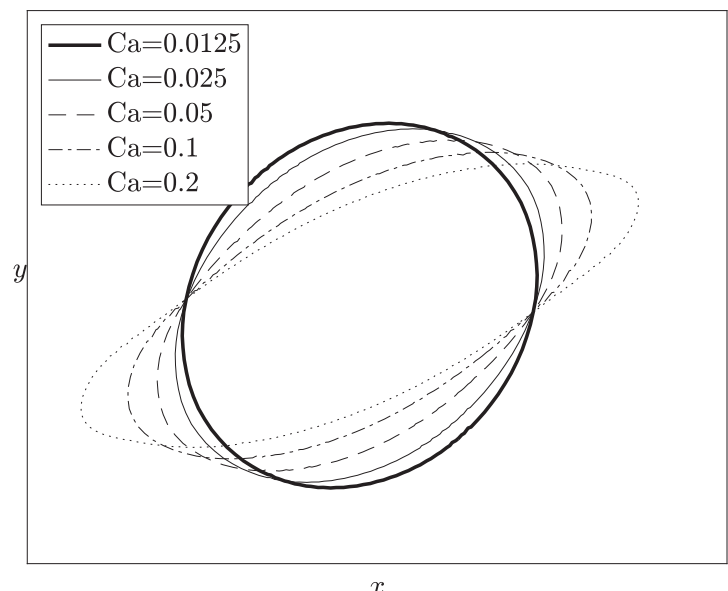

(a)

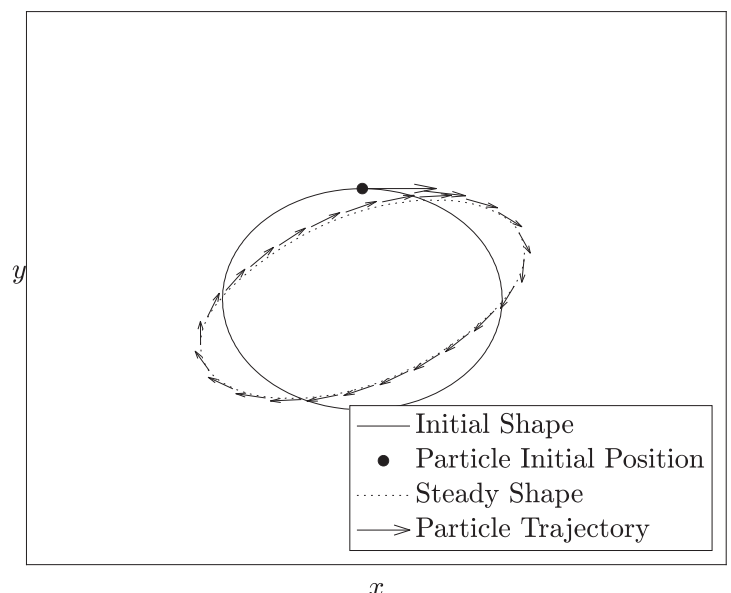

(b)

FIG. 7. (a) Steady-state shape of capsules for various values of Ca. (b) The trajectory of a particle for an initially spherical capsule with Ca $=0.05$.

the particle rotates around the capsule in the tank-tread motion discussed above.

In Fig. 8(a) the evolution of $D_{x y}$ is plotted for initially spherical capsules with varying values of the dimensionless bending modulus, $E_{b}$. The capillary number is fixed at $\mathrm{Ca}=$ 0.05 , the viscosity ratio is fixed at $V=1$, the spontaneous curvature is set at $c_{0}=0$. The capsules exhibit the same tank-treading behavior discussed above with close agreement between results from the present study and the results published by Le [42]. For a fixed capillary number, increasing $E_{b}$ results in reduced deformation.

In Fig. 9(a) the internal to external viscosity ratio is set to $V=5$ for varying values of the capillary number. The effects of the membrane's bending stiffness are ignored by setting $E_{b}=0$. Close agreement can be seen between the present results and those published by Le and Tan [25] and Ramanujan and Pozrikidis [12]. The capsules exhibit tank-treading behavior; however, the increased viscosity ratio results in reduced deformation of the capsule when compared with the deformation seen for $V=1$.

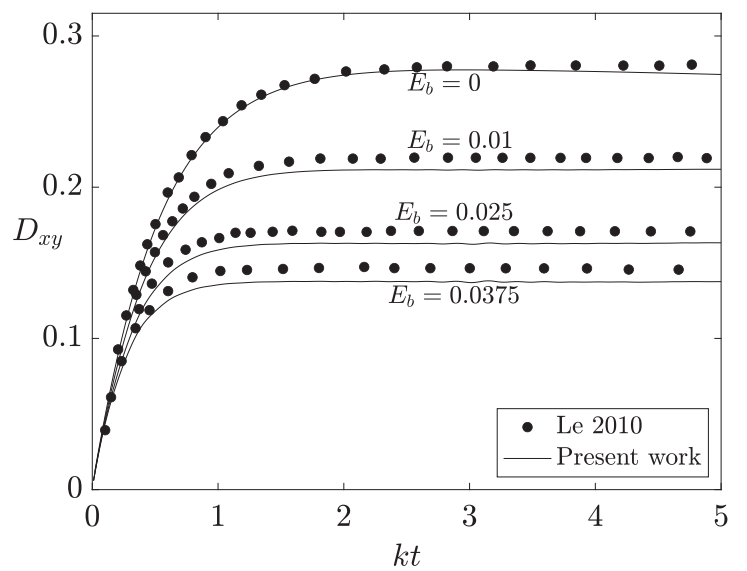

(a)
To assess the range of validity for the quasisteady approach proposed here, Fig. 10 compares $D_{x y}$ and $\theta / \pi$ for $\operatorname{Re}=0.05, \operatorname{Re}=0.1$, and $\operatorname{Re}=0.25$ using the standard LBM, which solves the time-dependent incompressible Navier-Stokes equations, and the quasisteady approach proposed here. In this figure the thin lines are the deformation parameter and the bold face lines are the inclination angle. Both simulations are conducted for spherical capsules with $\mathrm{Ca}=0.05, V=1$, and $E_{b}=0$ for a neo-Hookean membrane. In Fig. 10(a), for $\operatorname{Re}=0.05$, the two numerical schemes are seen to produce nearly equivalent results, suggesting that for $\mathrm{Re}=0.05$ neglecting the transient term of the Navier-Stokes equations does not affect the dynamics of the capsule deformation. In Figs. 10(b) and 10(c), the deformation parameter and inclination angles are seen to reach the same equilibrium values for both fluid solvers, which is unsurprising since the capsule's attainment of an equilibrium shape results in a steady flow pattern. Before reaching the equilibrium shape, however, the deformation and inclination of the quasisteady simulation can be seen to deviate from the standard LBM,

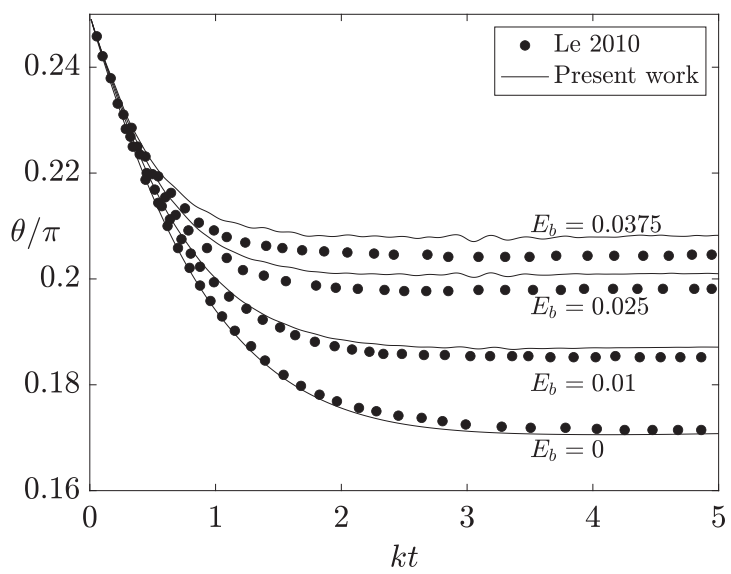

(b)

FIG. 8. (a) The deformation parameter and (b) inclination angle for initially spherical capsules with $\mathrm{Ca}=0.05$ and $V=1$ for various values of $E_{b}$. 


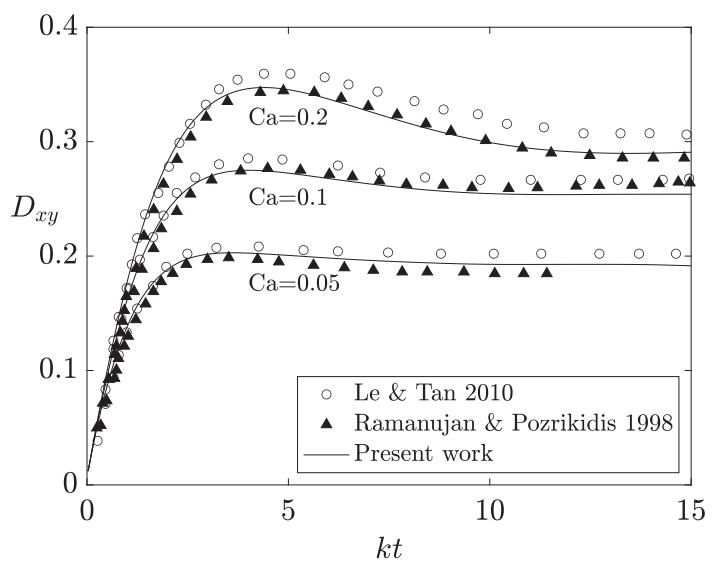

(a)

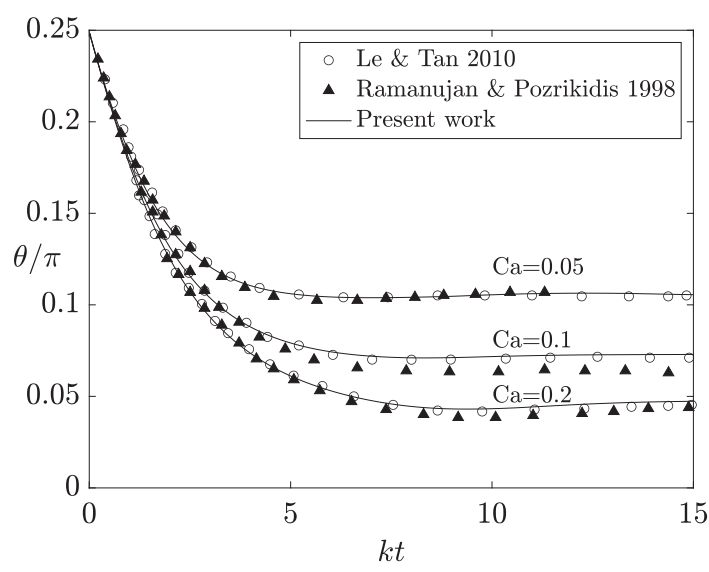

(b)

FIG. 9. (a) The deformation parameter and (b) inclination angle for initially spherical capsules $V=5$ and $E_{b}=0$ for various values of Ca.

with larger deviations occurring for the case with the larger Reynolds number, $\operatorname{Re}=0.25$. Based on these results, care should be taken when simulating the dynamics of phenomena with $\operatorname{Re} \geqslant 0.1$, as neglecting the transient term in the NavierStokes equations may affect the dynamics.

\section{Spheroid capsules}

To further demonstrate the efficacy of our method we consider the deformation of an oblate spheroid in shear flow. An oblate spheroid resembles a flattened sphere, with two axes of equal length and the length of the third axis less than that of the other two. The parametrization for an oblate spheroid can be given in terms of the azimuthal angle, $\phi$, and polar angle, $\theta$, as follows:

$$
x=R \sin \theta \cos \phi, \quad z=R \sin \theta \sin \phi, \quad y=R \frac{b}{a} \cos \theta .
$$

In Eq. (45) $R$ is used to adjust the radius so that the spheroid has a volume equivalent to the volume of a sphere with radius 1 .

Unlike initially spherical capsules, the spheroids do not reach a constant steady shape. Instead, the capsule's shape and inclination oscillate while the membrane rotates around the interior fluid. For small enough values of $\mathrm{Ca}$, the capsule's motion will transition from tank-treading to a tumbling motion $[9,58,59]$.
In Fig. $11 D_{x y}$ and $\theta / \pi$ are shown for a spheroid with $b / a=0.9$ for $V=1, E_{b}=0$ and capillary numbers, $\mathrm{Ca}=$ $0.05, \mathrm{Ca}=0.1$, and $\mathrm{Ca}=0.2$. These simulations have been conducted by a number of authors including [12,22,25,27,42]. Here, the results are plotted alongside those reported by Le and Tan [25]. Close agreement is observed between the present simulations and those in Ref. [25].

In Fig. $12 D_{x y}$ and $\theta / \pi$ are shown for a spheroid with $b / a=0.5$ for $V=1, E_{b}=0$ and capillary numbers, $\mathrm{Ca}=$ $0.05, \mathrm{Ca}=0.1$, and $\mathrm{Ca}=0.2$. For $\mathrm{Ca}=0.2$ the results are compared with those reported by Sui et al. [22], with close agreement seen between the two studies. For $\mathrm{Ca}=0.05$ and $\mathrm{Ca}=0.1$ Figure 12 provides a comparison between $D_{x y}$ and $\theta / \pi$ for two fluid solvers: the quasisteady method proposed in this work and the standard MRT lattice Boltzmann method. As can be seen in the figures, the results using the two fluid solvers are indistinguishable. This finding was true for all of the simulations presented in the present study, further suggesting that the quasisteady approach is valid for $\mathrm{Re}=0.05$.

In Figs. 13(a) and 13(b) the trajectory of a single Lagrangian node on the capsule membrane is plotted for a simulation up to $k t=20$ with $\mathrm{Ca}=0.05, V=1$ and $E_{b}=0$ for spheroids with $b / a=0.9$ and $b / a=0.5$, respectively. Each arrow represents the particles current velocity for the duration of the simulation. The particle completes more than one rotation around the capsule membrane during the simulation, illustrating the deviations in capsule shape for spheroid

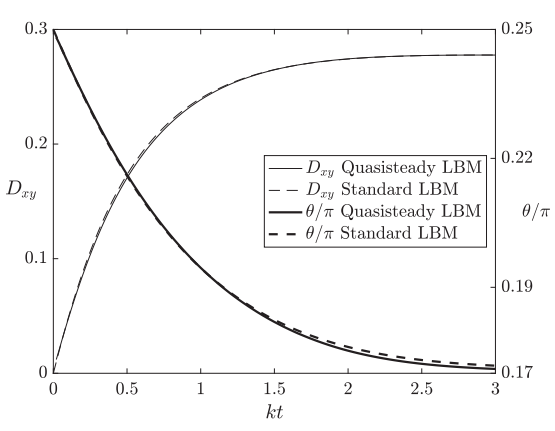

(a)

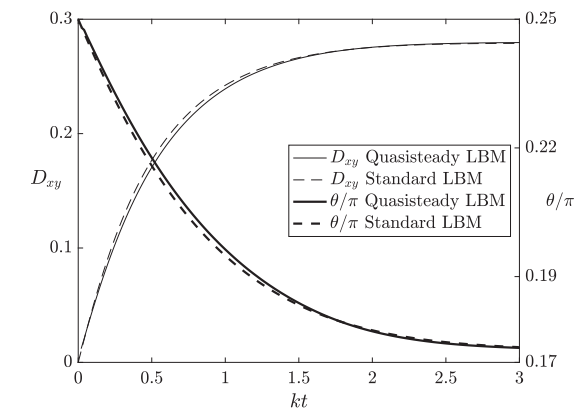

(b)

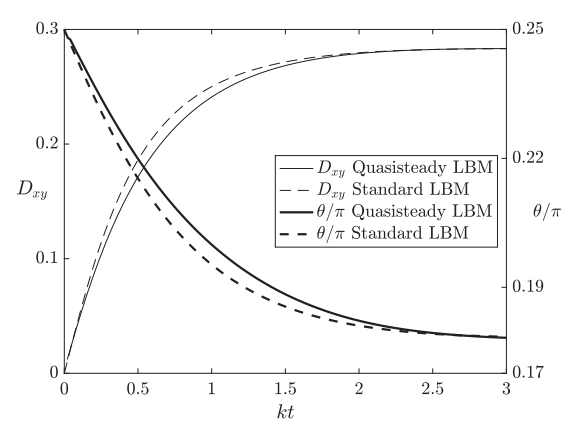

(c)

FIG. 10. (a) The deformation parameter and inclination angle for (a) $\operatorname{Re}=0.05$, (b) $\operatorname{Re}=0.1$, and (c) $\operatorname{Re}=0.25$. 


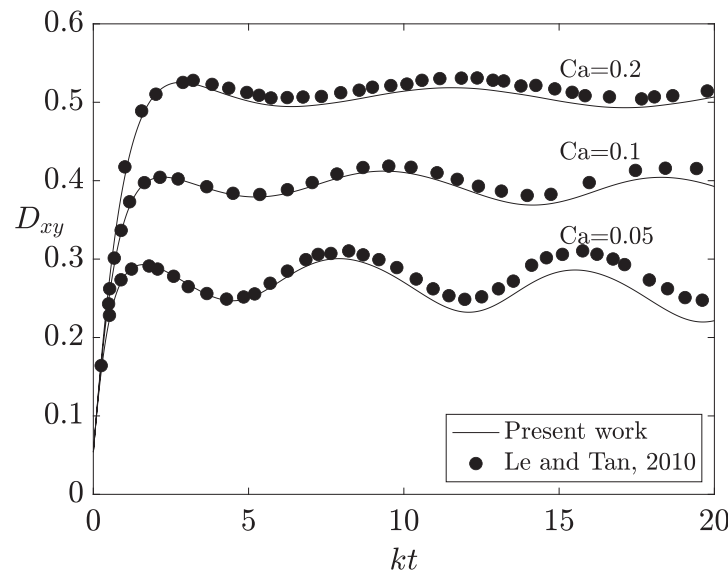

(a)

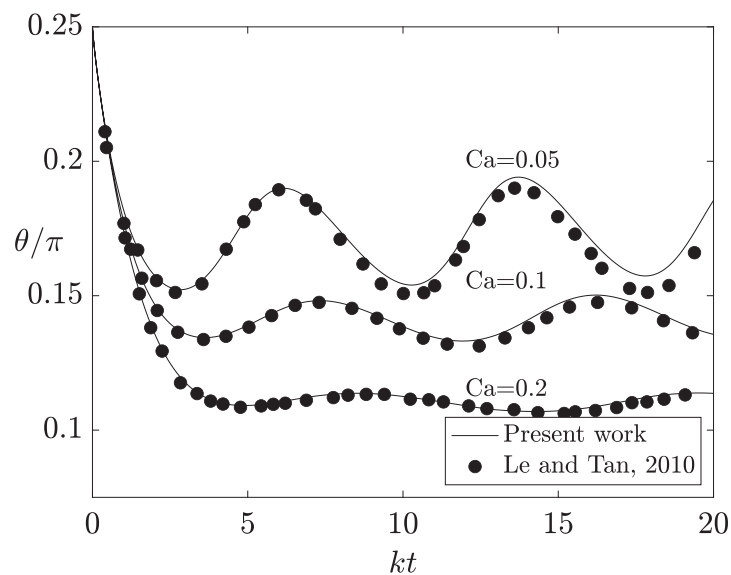

(b)

FIG. 11. (a) The deformation parameter and (b) inclination angle for various values of Ca for a spheroid with $b / a=0.9$.

capsules. In Fig. 13(a), the capsule with $b / a=0.9$, undergoes, slight changes in the shape as the particle revolves around the interior fluid. In Fig. 13(b) the capsule's shape and inclination angle oscillate more dramatically during tank treading. The larger deviations seen in Fig. 13(b) are due to the larger difference between the major and minor axis of the spheroid, indicated by the lower value of $b / a$.

\section{Biconcave capsules}

Next we consider the deformation of an initially biconcave capsule in shear flow. The parametrization for a biconcave capsule used here is given by Fung [60] in terms of the polar angle, $\theta$, and azimuthal angle, $\phi$, as

$$
\begin{aligned}
& x=a \alpha \sin \theta \cos \phi, \quad y=\frac{a \alpha}{2}\left(k_{0}+k_{1} \sin ^{2} \theta-k_{2} \sin ^{4} \theta\right) \cos \theta, \\
& z=a \alpha \sin \theta \sin \phi,
\end{aligned}
$$

where $\alpha=1.3858, k_{0}=0.207, k_{1}=2.003$, and $k_{2}=1.123$, and $a$ is the radius of a sphere with the same volume as the capsule. The resulting mesh can be seen in Fig. 3(b).
A variety of values of the spontaneous curvature, $c_{0}$, can be found in the literature. In Refs. [26,61] a constant negative value is used for the spontaneous curvature. We have followed $[20,62]$ and set the spontaneous curvature, $c_{0}$, in Eq. (31) to $c_{0}=-2 H_{0}$, where $H_{0}$ is the initial curvature of the capsule. This selection of the spontaneous curvature facilitates comparison between the present work and Refs. [20,62].

Although Refs. [20,62] used the same spontaneous curvature, they chose different values for $E_{b}$. In this work we use the values reported by Sinha and Graham [20], which are $E_{b}=0.03$ and $C=10$. The surface elasticity modulus, $G_{s}$, used in Ref. [20] corresponds to $E_{s}=2 G_{s}$, requiring us to rescale these quantities by $1 / 2$. Thus, in this work, the bending stiffness was held fixed at $E_{b}=0.015$ and Skalak's strain energy functional was used with a value of $C=5$, matching the values used in Ref. [20].

The dynamics of biconcave capsules in shear flow can be broadly grouped into two categories: a tumbling phase and a tank-treading phase. The tumbling phase typically occurs at low shear rates and high viscosity ratios. In the tumbling phase the capsule's shape remains roughly constant while the

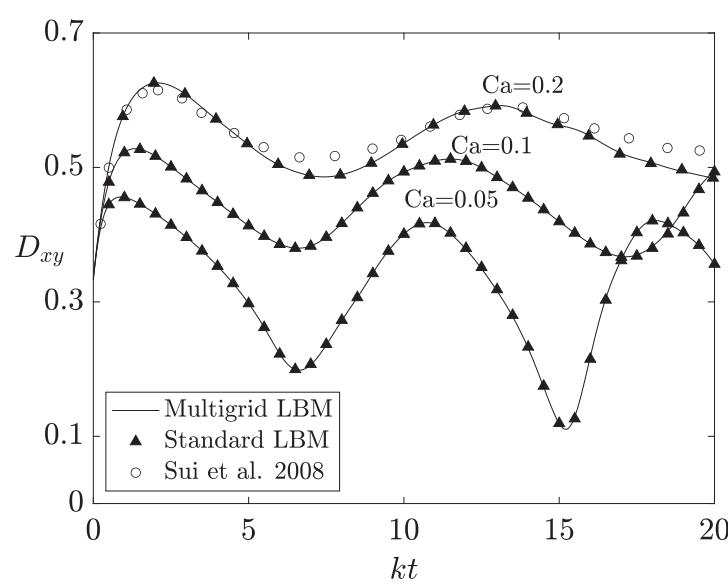

(a)

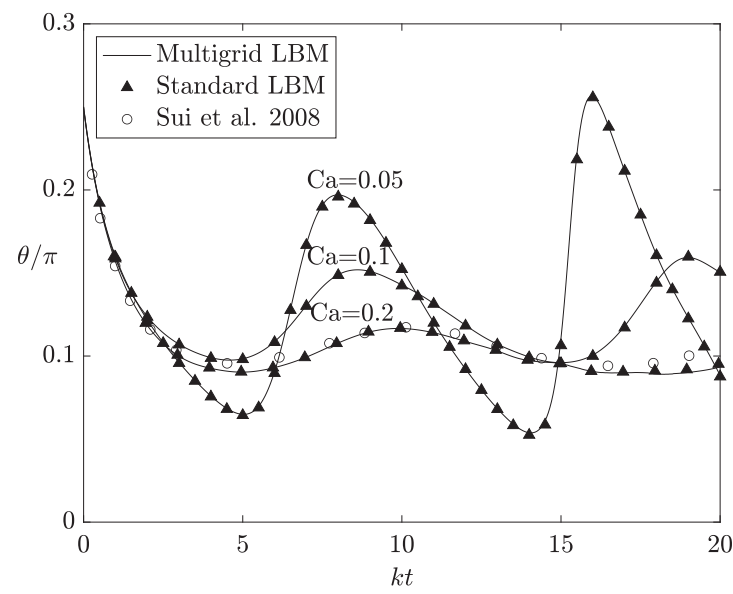

(b)

FIG. 12. (a) The deformation parameter and (b) inclination angle for various values of $C a$ for a spheroid with $b / a=0.5$. 


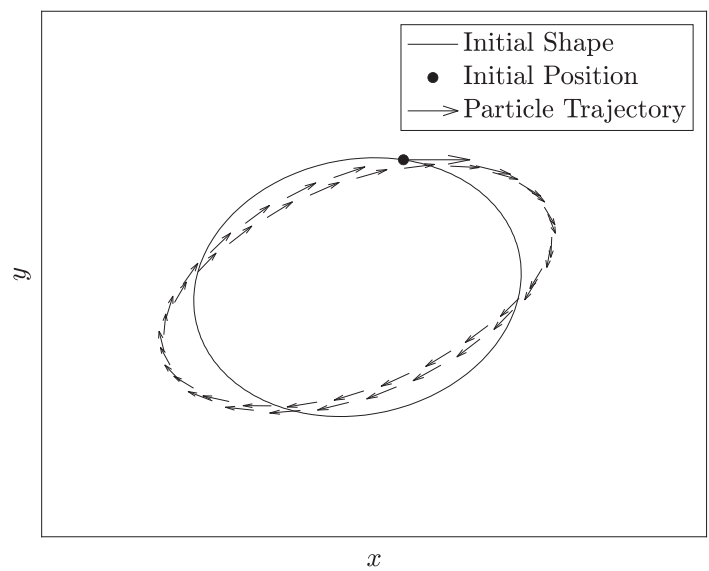

(a)

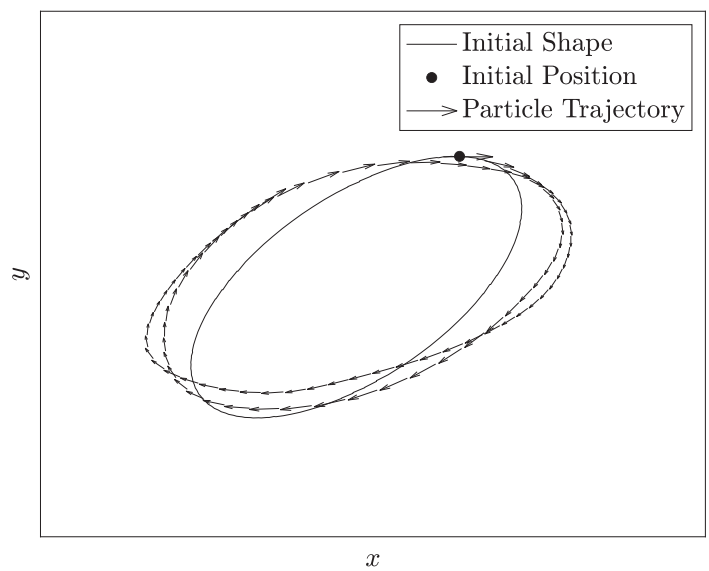

(b)

FIG. 13. (a) The trajectory of a particle on an initially spheroidal capsule with $b / a=0.9$ with $\mathrm{Ca}=0.05$. (b) The trajectory of a particle on an initially spheroidal capsule with $b / a=0.5$ with $\mathrm{Ca}=0.05$.

capsule undergoes rotations in the plane of shearing. Figure 14 shows the profile of an initially biconcave capsule with $E_{b}=$ $0.015, \mathrm{Ca}=0.25$, and $V=4$ undergoing the tumbling motion. As can be seen in the figure, the capsule retains the biconcave shape while rotating around in the center of the domain.

The tank-treading phase, which typically occurs at higher shear rates and lower viscosity ratios, is characterized by a rotation of the capsule membrane around the interior fluid. Figure 15 shows the profile of an initially biconcave capsule with $E_{b}=0.015, \mathrm{Ca}=0.75$, and $V=4$ undergoing the tanktreading motion. As can be seen, the shape and inclination angle remain roughly constant throughout the motion, while the membrane rotates around the interior fluid. In between these two phases are transition phases, where a capsule will oscillate between these two behaviors or exhibit more complicated dynamics that combine the two motions [18,20,26,28].

Figure 16 shows the inclincation angle for biconcave capsules in shear flow with $E_{b}=0.015, C=5$ and spontaneous curvature $c_{0}=-2 H_{0}$. Figure 16(a) illustrates the effect of increased capillary numbers. The viscosity ratio for the simulations in Fig. 16(a) was $V=4$. At $\mathrm{Ca}=0.25$ the capsule undergoes tumbling motion. At $\mathrm{Ca}=0.4$ the capsule first begins a tumbling motion, rotating $\pi$ radians before reaching a roughly constant inclination angle with a tank-treading motion. At $\mathrm{Ca}=0.75$ the capsule undergoes tank-treading motion.

Figure 16(b) illustrates the effect of increasing the viscosity ratio. The capillary number for the simulations in Fig. 16(b) are $\mathrm{Ca}=0.4$. At $V=2$ the capsule undergoes tank-treading motion, at $V=4$ the capsule is in a transition phase, and at $V=10$ the capsule undergoes tumbling motion. As can be seen, increasing the viscosity ratio has a similar effect to decreasing the capillary number.

A number of numerical $[20,26,61,62]$ and experimental [4] studies have been conducted on the motion of red blood cells as a function of the capillary number and viscosity ratio. To assess the fidelity of our model we ran simulations with viscosity ratios ranging from $V=0.5$ to $V=10$ and capillary numbers ranging from $\mathrm{Ca}=0.05$ to $\mathrm{Ca}=1$. The simulations were then categorized as undergoing a tank-treading phase, a tumbling phase or a transitional phase.

The results from these simulations can be seen in Fig. 17. The plot also includes the boundaries between the tanktreading and tumbling phases found by Sinha and Graham [20] and Le and Tan [62]. The boundaries between the different phases of motion exhibit close qualitative and quantitative agreement.

\section{COMPUTATIONAL EFFICIENCY}

To demonstrate the efficiency gains attained with the dual time-stepping strategy proposed in this work, we have conducted simulations for spherical and biconcave capsules for a variety of dimensionless parameters using the standard LBMIBM capsule model and the DTS method proposed in this work. The simulations were run on the Turing High Performance Computing Cluster at Old Dominion University using 2.3 GHz CPUs with $128 \mathrm{~GB}$ RAM. The resolution on the

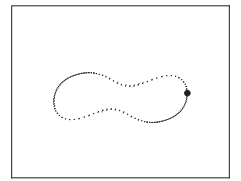

(a)

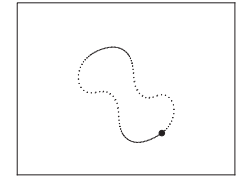

(b)

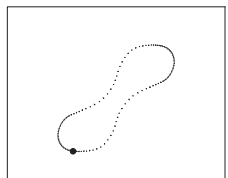

(c)

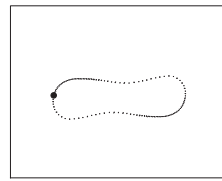

(d)

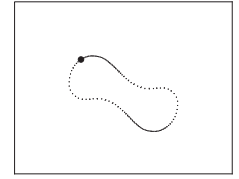

(e)

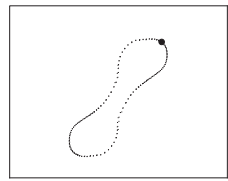

(f)

FIG. 14. The profile of an initially biconcave capsule at (a) $k t=5$, (b) $k t=8$, (c) $k t=11$, (d) $k t=15$, (e) $k t=18$, and (f) $k t=21$. The black dot is the location of a marker point on the membrane. 


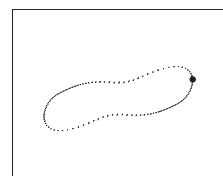

(a)

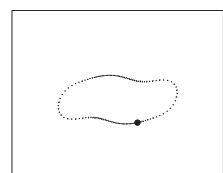

(b)

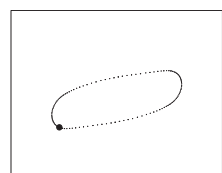

(c)

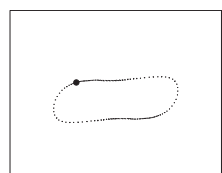

(d)

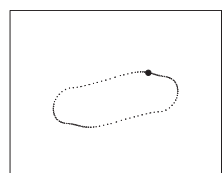

(e)

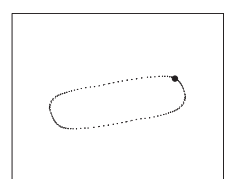

(f)

FIG. 15. The profile of an initially biconcave capsule at (a) $k t=4$, (b) $k t=10$, (c) $k t=16$, (d) $k t=22$, (e) $k t=28$, and (f) $k t=30$. The black dot is the location of a marker point on the membrane.

fine grid was $N=129$ in each dimension for both the single grid and multigrid simulations. The Eulerian domain used was $[0,12.8 a]^{3}$ for both simulations which, coupled with the resolution of $N=129$ in each direction, results in a time step of $\delta t=0.1 a \mathrm{~s}$ for the standard LBM simulation. As in Sec. VII, the simulations in this section are normalized by setting $\mathrm{Re}=$ $0.05, a=1 \mathrm{~m}$, and $\rho=1 \mathrm{~kg} \mathrm{~m}^{-3}$. The capsule mesh consists of 5120 triangular elements comprised of 2562 Lagrangian nodes. For the DTS scheme the time step was chosen using Eq. (43) and an iterative tolerance of $\epsilon=10^{-5}$ was used.

\section{A. Efficiency for spherical simulations}

To study the speedup offered by the newly proposed method we conduct simulations for spherical capsules using the standard LBM-IBM capsule model and the DTS scheme proposed here. The capsules were assumed to have neoHookean membranes with $V=1$ and $E_{b}=0$ with capillary numbers ranging from 0.00625 to 0.2 and shear rates ranging from $2 \times 10^{-4} \mathrm{~s}^{-1}$ to $1.6 \times 10^{-3} \mathrm{~s}^{-1}$. To facilitate comparison across capillary numbers, we alter the duration of the simulations based on the capillary number. For simulations with $\mathrm{Ca}=0.00625$ the simulations are run up to $k T=0.5$, for $\mathrm{Ca}=0.0125$ simulations are run up to $k T=1$, for $\mathrm{Ca}=$ 0.025 simulations are run up to $k T=2$, and for $\mathrm{Ca} \geqslant 0.05$ we simulate up to $k T=4$. This accounts for the smaller elastic timescales and smaller values of $k \Delta t$ used at smaller values of $\mathrm{Ca}$.

In Fig. 18(a) the speedup achieved by the DTS method is plotted against the shear rate. As can be seen in the figure, the speedup offered by the DTS method is proportional to $\mathrm{Ca}$ and inversely proportional to $k$. Both of these relationships can be understood by examining the time-step constraint for the DTS scheme given by Eq. (43). The dimensionless time step for the standard LBM, $k \delta t$, is determined by $k$ for a fixed value of $\mathrm{Ca}$, whereas for the DTS scheme the dimensionless physical time step, $k \Delta t$, varies with Ca for a fixed value of $k$.

The size of the physical time step, $\Delta t$, is a strong predictor of the potential speedup obtained by the DTS scheme. This relationship can be seen in Fig. 18(b), which plots the speedup against the ratio $\Delta t / \delta t$. As can be seen in the figure, when $\Delta t / \delta t=100$ a speedup of 2 is achieved with the DTS scheme. The speedup increases proportionally as $\Delta t / \delta t$ increases, with peak efficiencies on the order of 20 for larger values of $\mathrm{Ca}$. Based on this, the physical timescale and accuracy of the scheme should allow for increases of the physical time step by a factor of 100 or more for efficient application of the DTS scheme proposed here.

To demonstrate the limiting effects of the physical timescales and the accuracy requirements we have plotted the speedup achieved by the DTS method against the ratio, $\tau_{e} / \delta t$ using a logarithmic scale for the $\mathrm{x}$ axis in Fig. 18(c). The ratio $\tau_{e} / \delta t$ indicates the separation of scale between the physical timescales and the LBM time step. From Eq. (43) we see that for $\mathrm{Ca} \leqslant 0.05 \Delta t$ is limited by the elastic timescale of the problem, whereas for $\mathrm{Ca}=0.1$ and $\mathrm{Ca}=0.2$ the time step is limited by the desired accuracy of the simulation. This relationship is apparent in the figure, where the speedup curves for $\mathrm{Ca} \leqslant 0.05$ are nearly identical, while $\mathrm{Ca}=0.1$ and $\mathrm{Ca}=0.2$ have distinct speedup curves. From the figure we

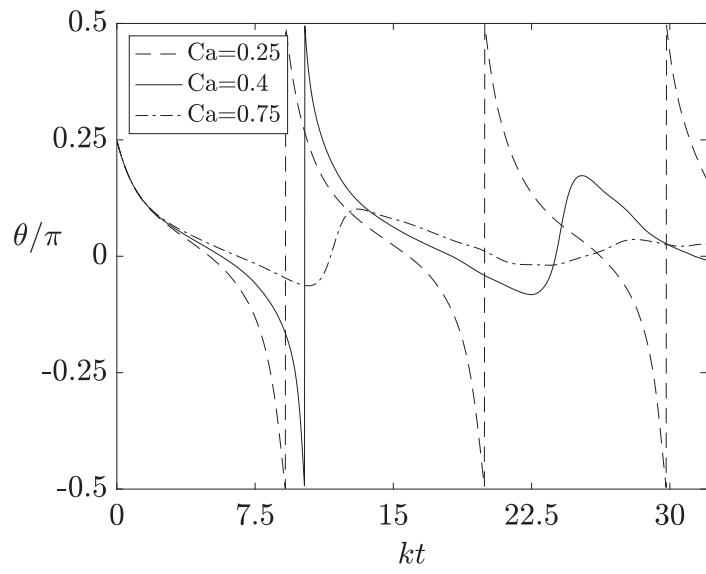

(a)

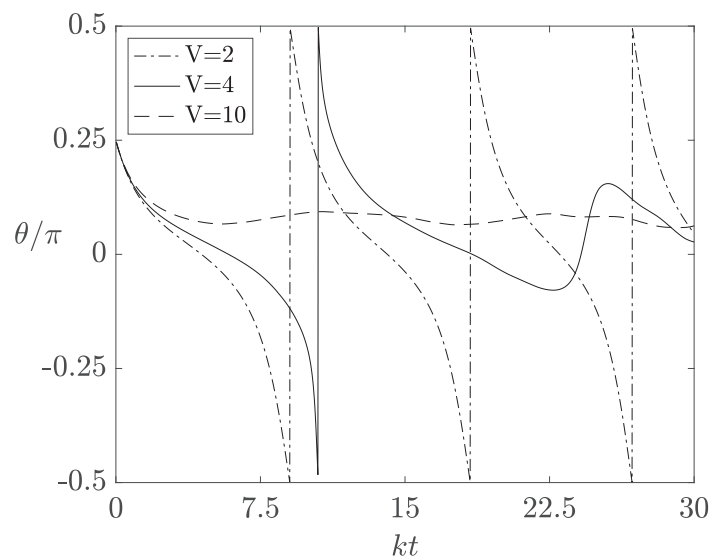

(b)

FIG. 16. (a) The inclination angle for capsules with various dimensionless shear rates. (b) The inclination angle for capsules with various viscosity ratios. 


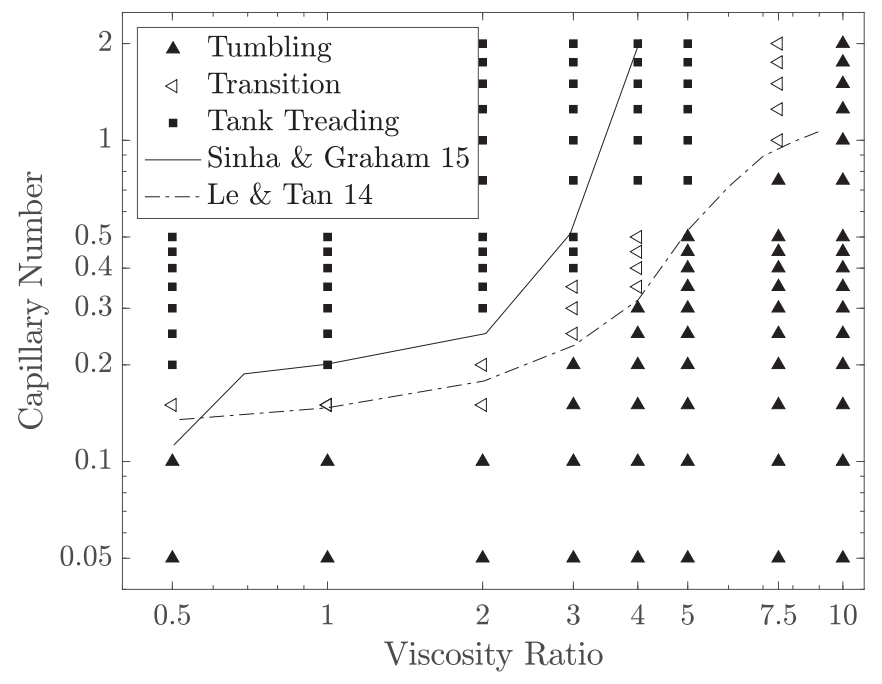

FIG. 17. Classification of simulations with biconcave capsules for a variety of capillary numbers and viscosity ratios.

recommend that $\tau_{e} / \delta t$ be on the order of 200 or more for efficient implementation of the DTS scheme proposed here.

While the speedup achieved using the DTS scheme diminishes as $k$ increases, for long simulations and nonspherical geometries $k$ is limited by the stability constraint in Eq. (39). Indeed, even for the spherical simulations discussed here $k>$ $1.2 \times 10^{-3} \mathrm{~s}^{-1}$ resulted in instability for some values of $\mathrm{Ca}$. It should also be mentioned that, since small values of $\mathrm{Ca}$ reach equilibrium in a shorter time frame, declines in efficiency with the DTS scheme for small values of $\mathrm{Ca}$ are not a major concern because the standard LBM-IBM capsule model can simulate these in a reasonable amount of time. The most dramatic speedup from the DTS scheme occur for $\mathrm{Ca} \geqslant 0.0125$ where simulation with the standard LBM-IBM capsule model can be much more expensive. In this regime, speedup between 4 and 20 times the standard LBM-IBM capsule model are achieved.

\section{B. Efficiency for biconcave simulations}

Simulating the dynamics of nonspherical capsules require much longer simulations because the capsules do not reach an equilibrium shape or flow field. These long simulations place more stringent constraints on the shear rate $k$, increasing the potential speedup offered by the DTS scheme. We find that $k \leqslant 2 \times 10^{-4} \mathrm{~s}^{-1}$ is required for simulations long enough to capture the dynamics of biconcave capsules. For the standard LBM-IBM capsule model this results in a dimensionless time step of $k \delta t=2 \times 10^{-5}$, which is far below the time step of the DTS scheme, which is set to $k \Delta t=0.02$ for the simulations in this section.

To study the efficiency gains for capsule simulations where the flow does not reach a steady state, we have run simulations with a biconcave capsule for each of the cases illustrated in Fig. 16(a). As in the figure, the viscosity ratio was set to $V=4$, the bending stiffness was set to $E_{b}=0.015$, and Skalak's modulus was set to $C=5$. Simulations were run up to $k t=30$ for $\mathrm{Ca}=0.25, \mathrm{Ca}=0.4$, and $\mathrm{Ca}=0.75$. These three cases were chosen to demonstrate the effect that the
TABLE I. CPU time comparison for a biconcave capsule with $V=4$ and $E_{b}=0.015$ for the cases shown in Fig. 16(a) run until $k t=30$

\begin{tabular}{|c|c|c|c|c|c|c|}
\hline & \multicolumn{2}{|c|}{$\mathrm{Ca}=0.25$} & \multicolumn{2}{|c|}{$\mathrm{Ca}=0.4$} & \multicolumn{2}{|c|}{$\mathrm{Ca}=0.75$} \\
\hline & LBM & mgLBM & LBM & mgLBM & LBM & mgLBM \\
\hline Time (s) & 322,339 & 43,693 & 322,373 & 16,661 & 322,423 & 9147 \\
\hline Efficiency & 1.0 & 7.4 & 1.0 & 19.3 & 1.0 & 35.2 \\
\hline
\end{tabular}

different capsule behaviors have on the computational efficiency of the model, as $\mathrm{Ca}=0.25$ results in a tumbling capsule, $\mathrm{Ca}=0.4$ results in a transient regime, and $\mathrm{Ca}=0.75$ results in a tank-treading capsule.

The results for these tests can be seen in Table I. As can be seen, the efficiency gains of the simulation improve as the capillary number increases. It should be noted that unlike the spherical case above, the relationship between $\mathrm{Ca}$ and CPU time is not related to $\Delta t$. As mentioned above, $k \Delta t=0.02$ for each value of $\mathrm{Ca}$ considered here. Instead the relationship between $\mathrm{Ca}$ and CPU time is due to the behavior of the capsule at the various capillary numbers as illustrated in Fig. 16(a).

In the tumbling regime at $\mathrm{Ca}=0.25$, the position of the capsule within the fluid changes at each time step. This alters the flow field near the capsule, requiring more iterations of the multigrid LBM algorithm at each time step. In the transient regime at $\mathrm{Ca}=0.4$, the capsule begins by tumbling before oscillating slightly around $\theta=0$ with minimal deformation. Thus, after the tumbling phase is completed and changes in the capsule's inclination angle are reduced, variations in the flow field decrease allowing faster convergence with the multigrid LBM at each time step. Finally, in the tank-treading regime at $\mathrm{Ca}=0.75$, the capsule's angle remains roughly constant as the membrane rotates around the capsule. Thus, after reaching the steady shape, variation in the flow field is reduced between time steps, requiring fewer iterations of the fluid solver at each time step.

\section{DISCUSSION}

In this work a quasisteady, dual time-stepping lattice Boltzmann method was presented for flow-induced capsule deformation simulations using the immersed boundary method. The method was validated by comparing the results to those reported in previous studies for a wide range of dimensionless parameters and capsule geometries. Separating the LBM time step from the capsule time-step results in simulations on the order of 10 times faster than those achieved by the standard LBM-IBM capsule model. Furthermore, de-coupling the physical time step from the LBM time step allows us to choose the physical time step based on the stability constraints of the elastic problem, without consideration for the LBM scheme.

The proposed method treats the flow as a quasisteady problem and thus the method is limited to flows in the low Reynolds number regime. To ensure physical fidelity, careful consideration of the expected dynamics is required when using this numerical scheme for $\mathrm{Re} \geqslant 0.1$. For phenomena where the quasisteady assumption does not hold, the method 


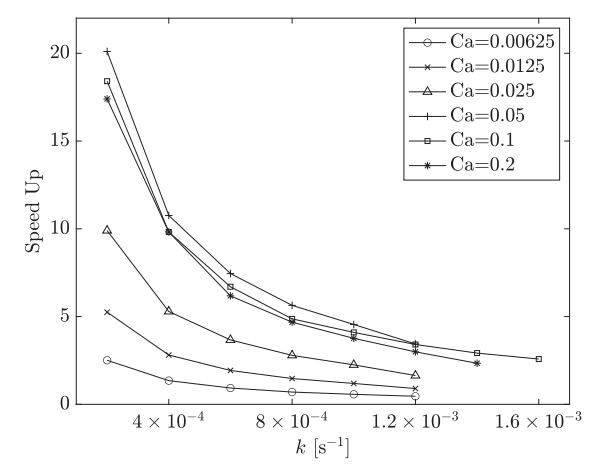

(a)

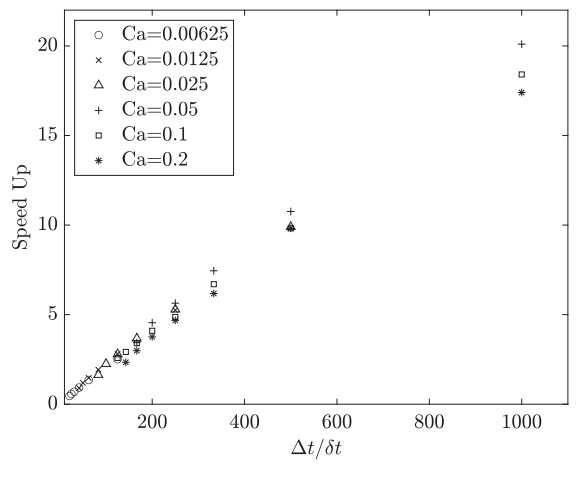

(b)

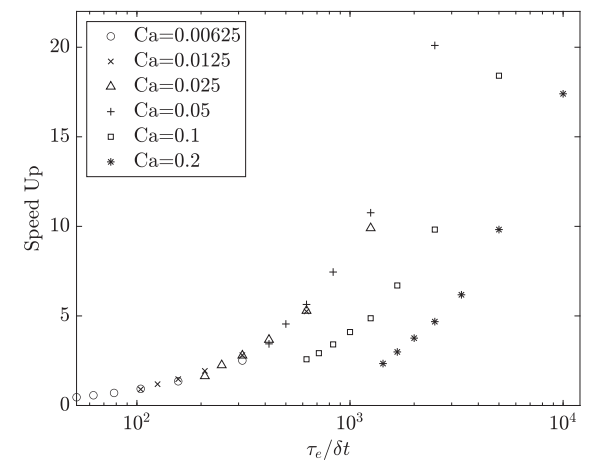

(c)

FIG. 18. (a) The speedup as a function of the shear rate, $k$. (b) The speedup as a function of the ratio $\Delta t / \delta t$. (c) The speedup as a function of the ratio $\tau_{e} / \delta t$.

proposed by Gsell et al. [38] could be employed. In the low Reynolds number regime discussed in this work, the proposed method has some advantages over the method from Ref. [38]. Neglecting the transient term reduces the memory footprint of the algorithm by avoiding storage of the previous velocity and density fields. The present method also employs the standard LBM procedures for the collision and streaming steps, allowing for quick implementation into current LBM-IBM capsule codes.

For efficient application of the DTS scheme the physical time step should be several orders of magnitude larger than the tradition LBM time step to defray the additional computational cost per time step. We find that the DTS scheme is twice as fast as the LBM scheme when a physical time step 100 times larger than the LBM time step is used. Larger differences between the two time steps increase the speedup of the DTS scheme proportionally. For a given application the suitability of the proposed scheme is related to the ratio of the physical timescale to the LBM time step. For elastic capsule simulations the minimum ratio of $\tau_{e} / \delta t$ for a speedup of 2 using the DTS scheme is approximately 200.
For the parameter regime in which standard LBM-IBM models of capsule deformation are most computationally expensive, the DTS scheme presented in this work results in speedup on the order of 20. Even for parameter regimes in which the increasing the physical time step is limited by the separation of scales we find speedup on the order of 4-10 times the standard LBM-IBM capsule. Future directions could include the consideration of additional membrane forces such as those arising from the membrane's viscosity or those exerted on the membrane by an electrostatic field. The development of a more efficient iterative solver or a refined residual equation could also offer potential improvements to the fluid algorithm presented in this work.

\section{ACKNOWLEDGMENTS}

Support from National Science Foundation Grant No. DMS-1319078 is gratefully acknowledged. This research was supported by the Turing High Performance Computing cluster at Old Dominion University.
[1] H. Schmid-Schönbein and R. Wells, Fluid drop-like transition of erythrocytes under shear, Science 165, 288 (1969).

[2] H. Goldsmith and J. Marlow, Flow behavior of erythrocytes I: Rotation and deformation in dilute suspensions, Proc. R. Soc. London B 182, 351 (1972).

[3] J. Dupire, M. Socol, and A. Viallat, Full dynamics of a red blood cell in shear flow, Proc. Nat. Acad. Sci. U.S.A. 109, 20808 (2012).

[4] T. Fischer and R. Korzeniewski, Threshold shear stress for the transition between tumbling and tank-treading of red blood cells in shear flow: Dependence on the viscosity of the suspending medium, J. Fluid Mech. 736, 351 (2013).

[5] D. Barthés-Biesel, Motion of a spherical microcapsule freely suspended in a linear shear flow, J. Fluid Mech. 100, 831 (1980).

[6] D. Barthés-Biesel and J. Rallison, The time-dependent deformation of a capsule freely suspended in a linear shear flow, J. Fluid Mech. 113, 251 (1981).
[7] S. Keller and R. Skalak, Motion of a tank-treading ellipsoidal particle in shear flow, J. Fluid Mech. 120, 27 (1982).

[8] J. M. Skotheim and T. W. Secomb, Red Blood Cells and Other Nonspherical Capsules in Shear Flow: Oscillatory Dynamics and the Tank-Treading-To-Tumbling Transition, Phys. Rev. Lett. 98, 078301 (2007).

[9] M. Abkarian, M. Faivre, and A. Viallat, Swinging of Red Blood Cells Under Shear Flow, Phys. Rev. Lett. 98, 188302 (2007).

[10] C. Pozrikidis, Finite deformation of liquid capsules enclosed by elastic membranes in simple shear flow, J. Fluid Mech. 297, 123 (1995).

[11] C. Pozrikidis, Effect of membrane bending stiffness on the deformation of capsules in simple shear flow, J. Fluid Mech. 440, 269 (2001).

[12] S. Ramanujan and C. Pozrikidis, Deformation of liquid capsules enclosed by elastic membranes in simple shear flow: Large deformations and the effect of fluid viscosities, J. Fluid Mech. 361, 117 (1998). 
[13] C. Peskin, The immersed boundary method, Acta Numerica 11, 479 (2002).

[14] C. Eggleton and A. Popel, Large deformation of red blood cell ghosts in a simple shear flow, Phys. Fluids 10, 1834 (1998).

[15] T. Omori, T. Ishikawa, D. Barthés-Biesel, A.-V. Salsac, Y. Imai, and T. Yamaguchi, Tension of red blood cell membrane in simple shear flow, Phys. Rev. E 86, 056321 (2012).

[16] A. Farutin, T. Biben, and C. Misbah, 3D numerical simulations of vesicle and inextensible capsule dynamics, J. Comput. Phys. 275, 539 (2014).

[17] Z. Peng, A. Mashayekh, and Q. Zhu, Erythrocyte responses in low-shear-rate flows: Effects of nonbiconcave stress-free state in the cytoskeleton, J. Fluid Mech. 742, 96 (2014).

[18] K. Tsubota, S. Wada, and H. Liu, Elastic behavior of a red blood cell with the membranes nonuniform natural state: Equilibrium shape, motion transition under shear flow, and elongation during tank-treading motion, Biomechan. Mod. Mechanobiology 13, 735 (2014).

[19] Z. Peng, S. Salehyar, and Q. Zhu, Stability of the tank-treading modes of erythrocytes and its dependence on cytoskeleton reference states, J. Fluid Mech. 771, 449 (2015).

[20] K. Sinha and M. D. Graham, Dynamics of a single red blood cell in simple shear flow, Phys. Rev. E 92, 042710 (2015).

[21] X. Li and K. Sarkar, Front tracking simulation of deformation and buckling instability of a liquid capsule enclosed by an elastic membrane, J. Comput. Phys. 227, 4998 (2008).

[22] Y. Sui, Y. Chew, P. Roy, and H. Low, A hybrid method to study flow-induced deformation of three-dimensional capsules, J. Comput. Phys. 227, 6351 (2008).

[23] Y. Sui, Y. Chew, P. Roy, Y. Cheng, and H. Low, Dynamic motion of red blood cells in simple shear flow, Phys. Fluids 20, 112106 (2008).

[24] D. V. Le, Effect of bending stiffness on the deformation of liquid capsules enclosed by thin shells in shear flow, Phys. Rev. E 82, 016318 (2010).

[25] D. Le and Z. Tan, Large deformation of liquid capsules enclosed by thin shells immersed in the fluid, J. Comput. Phys. 229, 4097 (2010).

[26] A. Z. K. Yazdani and P. Bagchi, Phase diagram and breathing dynamics of a single red blood cell and a biconcave capsule in dilute shear flow, Phys. Rev. E 84, 026314 (2011).

[27] W. Huang, C. Chang, and H. Sung, Three-dimensional simulation of elastic capsules in shear flow by the penalty immersed boundary method, J. Comput. Phys. 231, 3340 (2012).

[28] D. Cordasco and P. Bagchi, Intermittency in red blood cell dynamics, J. Fluid Mech. 759, 472 (2014).

[29] D. Mavriplis, Multigrid solution of the steady-state lattice Boltzmann equation, Comput. Fluids 35, 793 (2006).

[30] T. Krüger, F. Varnik, and D. Raabe, Efficient and accurate simulations of deformable particles immersed in a fluid using a combined immersed boundary lattice Boltzmann finite element method, Comput. Math. Appl. 61, 3485 (2011).

[31] J. Gounley and Y. Peng, Response and recovery times of elastic and viscoelastic capsules in shear flow, Commun. Comput. Phys. 17, 1151 (2015).

[32] J. Charrier, S. Shrivastava, and R. Wu, Free and constrained inflation of elastic membranes in relation to thermoformingnonaxisymmetric problems, J. Strain Analysis 24, 55 (1989).

[33] W. Helfrich, Elastic properties of lipid bilayers: Theory and possible experiments, Z. Naturforsch 28, 693 (1973).
[34] Ou-Yang Zhong-can and W. Helfrich, Bending energy of vesicle membranes: General expressions for the first, second, and third variation of the shape energy and applications to spheres and cylinders, Phys. Rev. A 39, 5280 (1989).

[35] J. Zhang, P. C. Johnson, and A. S. Popel, An immersed boundary lattice Boltzmann approach to simulate deformable liquid capsules and its application to microscopic blood flows, Phys. Biol. 4, 285 (2007).

[36] A. Ghaffarian and K. Hejranfar, An implicit dual time-stepping pectral dfifference lattice Boltzmann method for simulation of viscous compressible flows on structured meshes, Meccanica 54, 1561 (2019).

[37] G. Guzel and I. Koc, Time-accurate flow simulations using a finite-volume based lattice Boltzmann flow solver with dual time-stepping scheme, Inte. J. Comput. Meth. 13, 1650035 (2016).

[38] S. Gsell, U. D’Ortona, and J. Favier, Multigrid dual timestepping lattice Boltzmann method, Phys. Rev. E 101, 023309 (2020).

[39] K. Chang and W. Olbricht, Experimental studies of the deformation and breakup of a synthetic capsule in steady and unsteady simple shear flow, J. Fluid Mech. 250, 609 (1993).

[40] A. Walter, H. Rehage, and H. Leonhard, Shear induced deformation of microcapsules: Shape oscillations and membrane folding, Colloids Surfaces A 183-185, 123 (2001).

[41] E. Lac, D. Barthés-Biesel, N. Pelekasis, and J. Tsamopoulos, Spherical capsules in three-dimensional unbounded stokes flows: Effect of the membrane constitutive law and onset of buckling, J. Fluid Mech. 516, 303 (2004).

[42] D. V. Le, Subdivision elements for large deformation of liquid capsules enclosed by thin shells, Comput. Meth. Appl. Mech. Eng. 199, 2622 (2010).

[43] G. Taylor, The formulation of emulsions in definable fields of flow, Proc. R. Soc. 146, 501 (1934).

[44] D. d'Humiéres, I. Ginzburg, M. Krafczyk, P. Lallemand, and L. Luo, Multiple-relaxation-time lattice Boltzmann models in three dimensions, Phil. Trans. R. Soc. A: Math. Phys. Eng. Sci. 360, 437 (2002).

[45] P. Lallemand, L. Luo, and Y. Peng, A lattice Boltzmann fronttracking method for interface dynamics with surface tension $n$ two dimensions, J. Comput. Phys. 226, 1367 (2007).

[46] D. Patil, K. N. Premnath, and S. Banerjee, Multigrid lattice Boltzmann method for accelerated solution of elliptic equations, J. Comput. Phys. 265, 172 (2014).

[47] R. Skalak, A. Tozeren, R. Zarda, and S. Chien, Strain energy functions of red blood cell membranes, Biophys. J. 13, 245 (1973).

[48] D. Barthés-Biesel, A. Diaz, and E. Dhenin, Effect of constitutive laws for two-dimensional membranes on flow-induced capsule deformation, J. Fluid Mech. 460, 211 (2002).

[49] A. Guckenberg, M. Schrame, P. G. Chen, M. Leonetti, and S. Gekle, On the bending algorithms for soft objects in flows, Comput. Phys. Commun. 207, 1 (2016).

[50] A. Guckenberg and S. Gekle, Theory and algorithms to compute Helfrich bending forces: A review, J. Phys.: Condens. Matter 29, 203001 (2017).

[51] R. Garimella and B. Swartz, Curvature estimation for unstructured triangulations of surfaces, Tech. Rep., LA-UR-03-8240, 2003. 
[52] M. Reuter, S. Biasotti, D. Giorgi, G. Patané, and M. Spagnuolo, Discrete laplace-beltrami operators for shape analysis and segmentation, Comput. Graphics 33, 381 (2009).

[53] U. Pinkall and K. Polthier, Computing discrete minimal surfaces and their conjugates, Exper. Math. 2, 15 (1993).

[54] J. Walter, A.-V. Salsac, D. Barthés-Biesel, and P. L. Tallec, Coupling of finite element and boundary integral methods for a capsule in a stokes flow, Int. J. Numer. Methods Eng. 83, 829 (2010).

[55] J. Walter, A.-V. Salsac, and D. Barthés-Biesel, Ellipsoidal capsules in simple shear flow: Prolate versus oblate initial shapes, J. Fluid Mech. 676, 318 (2011).

[56] D. Barthés-Biesel, Motion and deformation of elastic capsules and vesicles in flow, Ann. Rev. Fluid Mech. 48, 25 (2016).
[57] C. Armstrong and Y. Peng, An mrt extension to the multigrid lattice Boltzmann method, Commun. Comput. Phys. 26, 1178 (2019).

[58] S. Kessler, R. Finken, and U. Seifert, Swinging and tumbling of elastic capsules in shear flow, J. Fluid Mech. 605, 207 (2008).

[59] P. Bagchi and R. M. Kalluri, Dynamics of nonspherical capsules in shear flow, Phys. Rev. E 80, 016307 (2009).

[60] Y. Fung, Biomechanics: Mechanical Properties of Living Tissues (Springer-Verlag, Berlin, 1993).

[61] D. Cordasco, A. Yazdani, and P. Bagchi, Comparison of erythrocyte dynamics in shear flow under different stress-free configurations, Phys. Fluids 26, 041902 (2014).

[62] D. Le and Z. Tan, Hydrodynamic interaction of elastic capsules in bounded shear flow, Commun. Comput. Phys. 16, 1031 (2014). 